
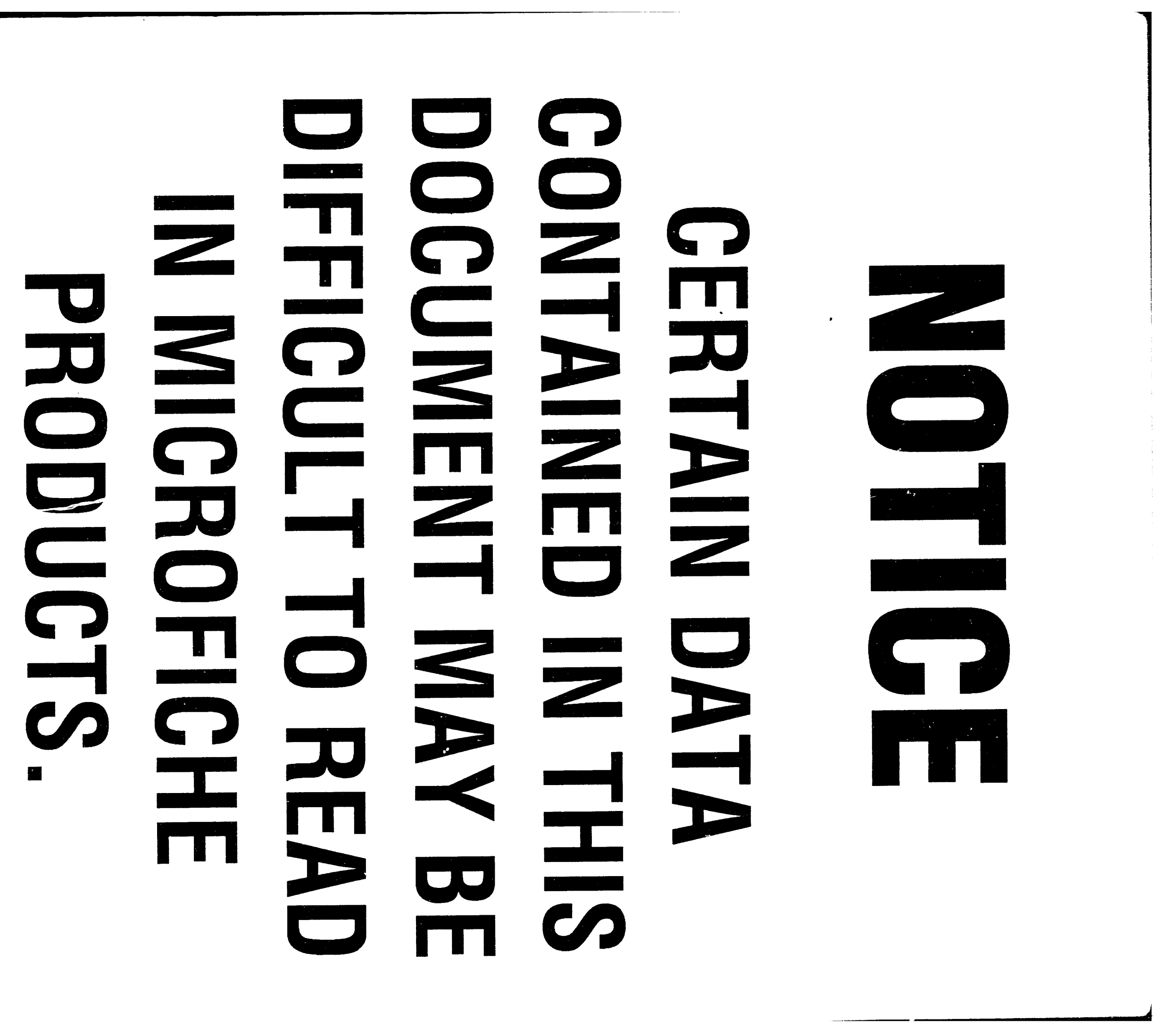


\section{REGIONAL SURVEY MONTHLIES 1948}

W. $C$. Berlin, Jr.

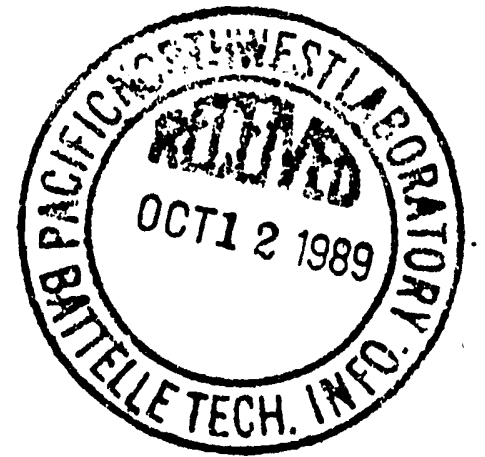

\section{DISCLAIMER}

This report was prepared as an account of work sponsored by an agency of the United States Government. Neither the United States Government nor any agency thereof, nor any of their employees, makes any warranty, express or implied, or assumes any legal liability or responsibility for the accuracy, completeness, or usefulness of any information, apparatus, product, or process disclosed, or represents that its use would not infringe privately owned rights. Reference herein to any specific commercial product, process, or service by trade name, trademark, manufacturer, or otherwise does not necessarily constitute or imply its endorsement, recommendation, or favoring by the United States Government or any agency thereof. The views and opinions of authors expressed herein do not necessarily state or reflect those of the United States Government or any agency thereof.

REPRODUCED EROM BEST

AVAILABLE COPY

UNCLASS IFIED

$48 \operatorname{lin}^{4}$

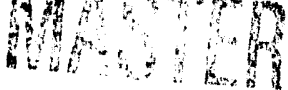




$$
\because \ldots+C a
$$

$$
1+\dot{w}-89074
$$

\section{$0+30,1948$}

$\therefore$ Wasinqlevich

rians: W.C. Berlin J.

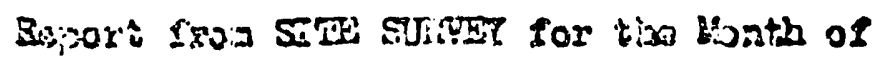

$$
\text { Octobr- } 1948
$$

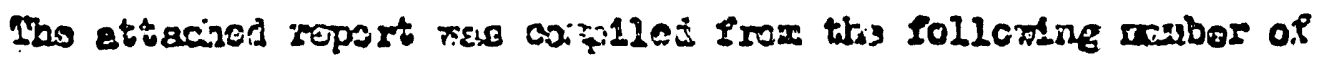
ast 250

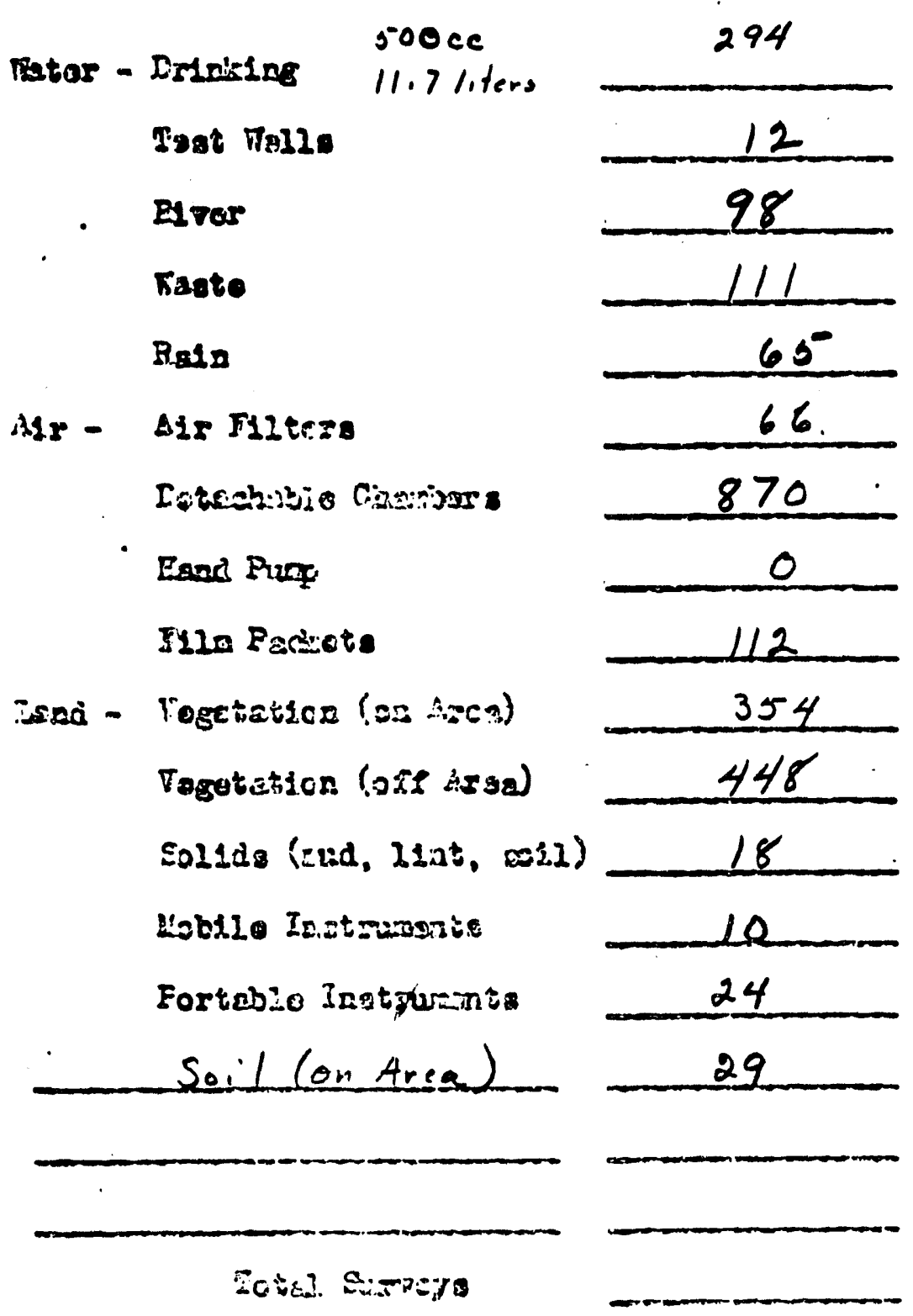


Tringision -

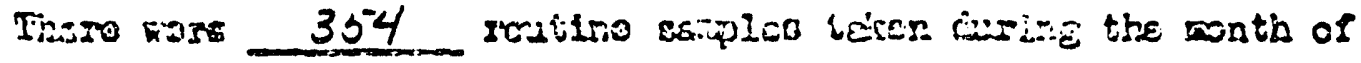

There has keed very lettle ehangs in the level ef ceatomingtion

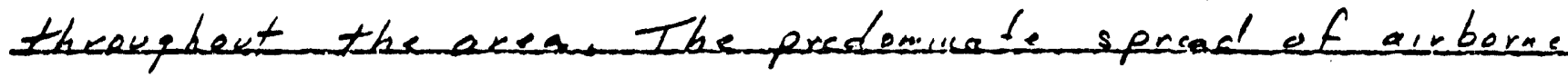
contanuation is mexe endent in the Pesente cenuel eres.

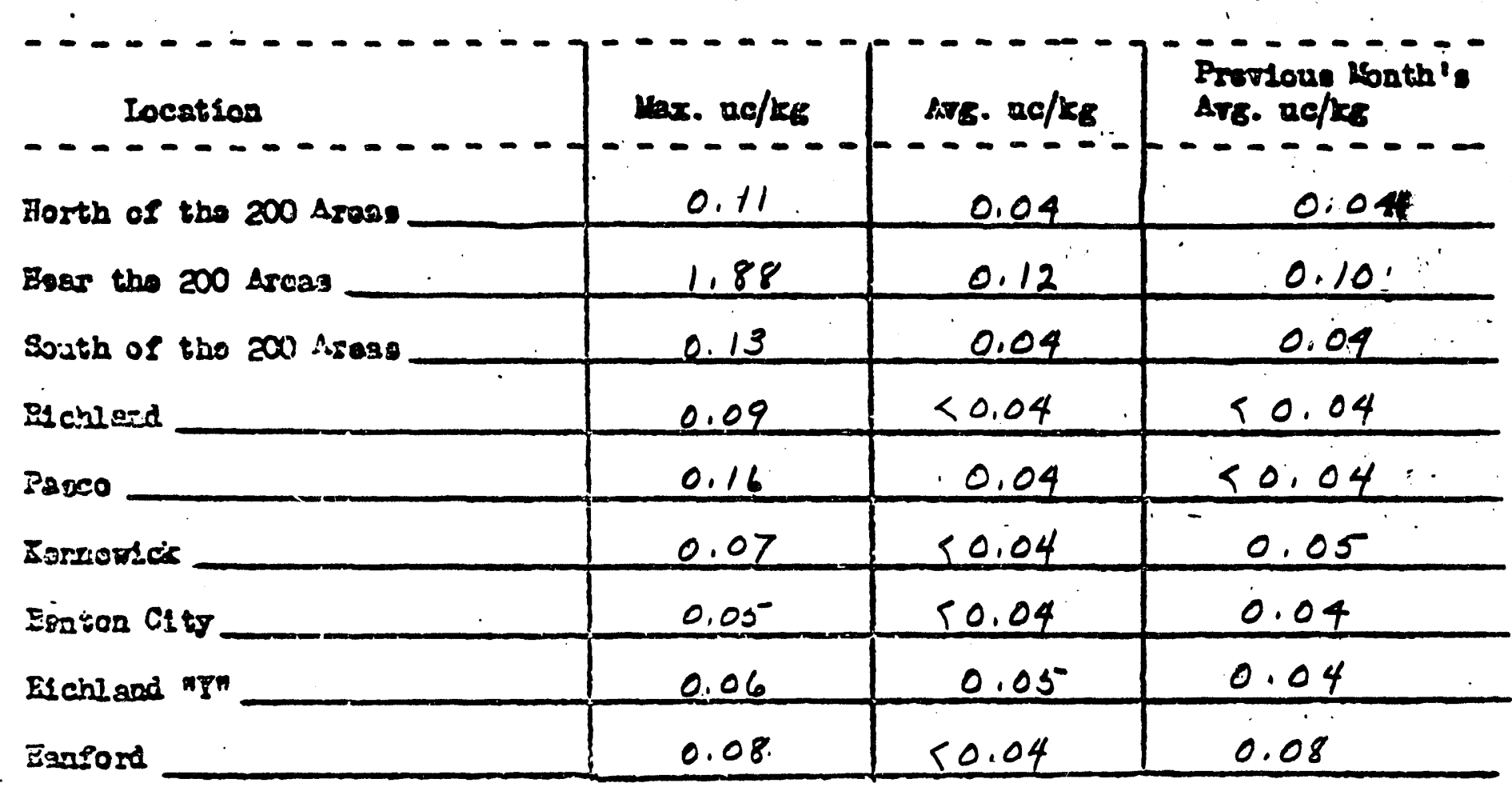

\section{Inetdo Sejuagen Astan}

\section{I00:100}

200 Fiøet

200 sias $\because=5$. es/tso

\begin{tabular}{|c|}
\hline$=0.05 / 55$ \\
\hline 0.43 \\
\hline 10.05 \\
\hline 10.50 \\
\hline
\end{tabular}

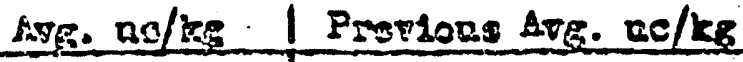

$0.08 \quad 0.09$

0.24

1

* Ihis activity was due to a which

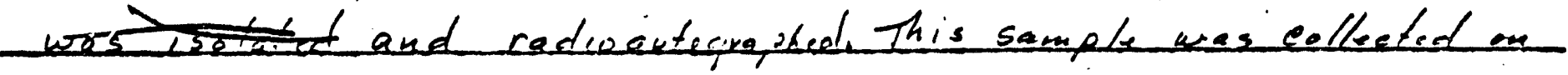




\section{Er:s= miremi. .}

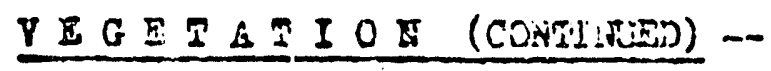

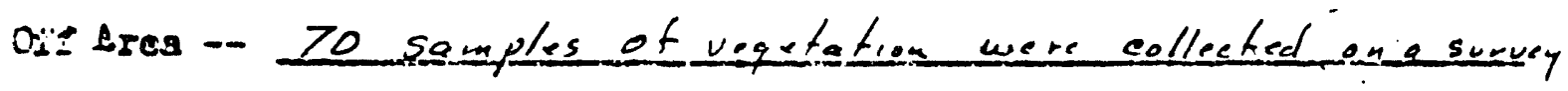

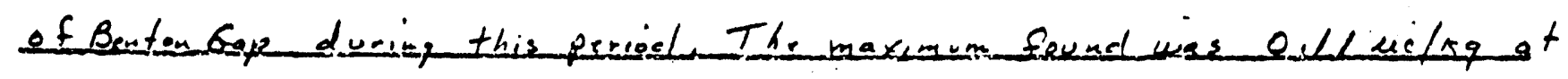

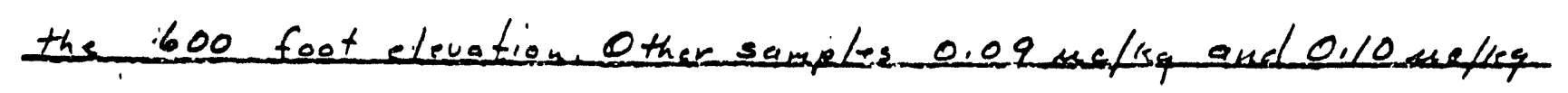
were taken at 2000 feet and 1900 footespecturly the averace of all Semples was $<0.04$ mels 7

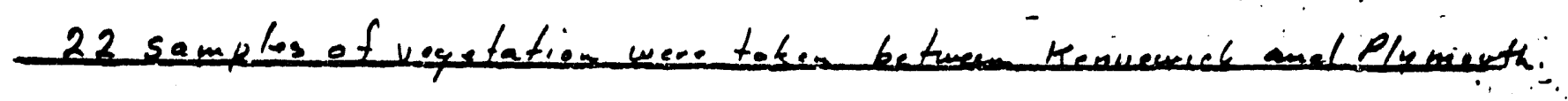

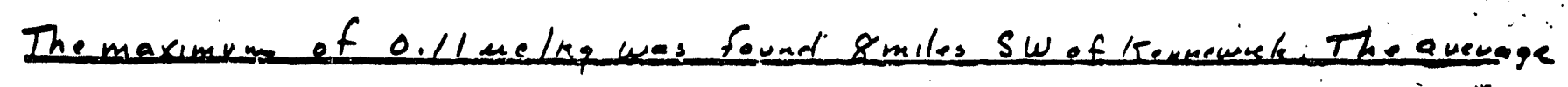
of all sameles was sopor walso

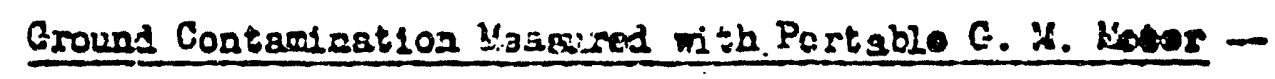

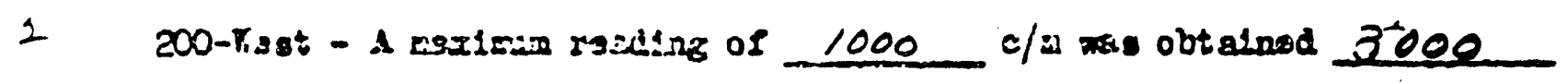
fost SE. of the E⿰氵工⿺ Stact.

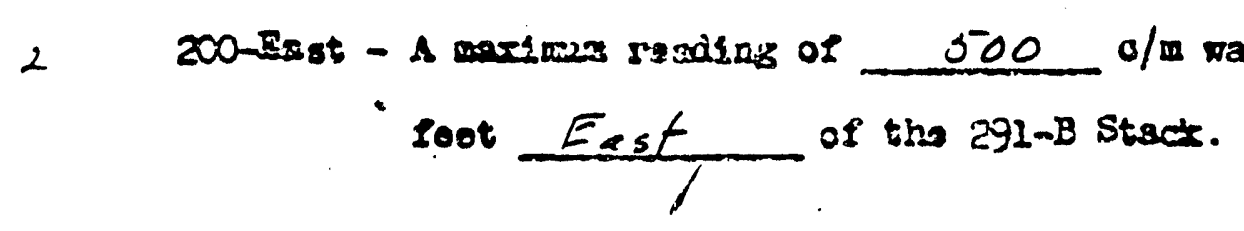

$\underline{\text { Soil }}=29$ samples of soil were collected during this

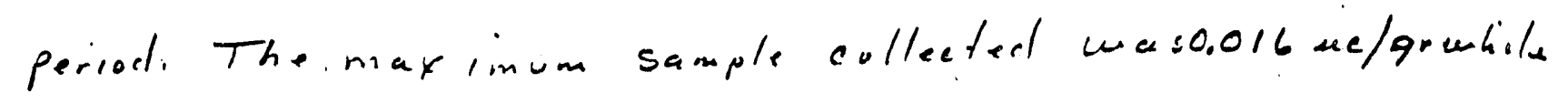
the average of all samplos was $0.012 \mathrm{me} / \mathrm{qm}$. 


$$
\text { Exis } \cos
$$

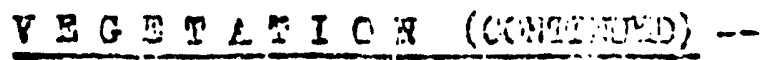

OiY Ares - Theremene four trips of fares this perod.

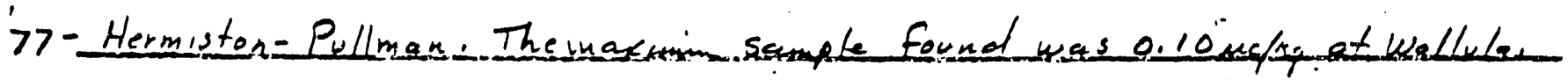

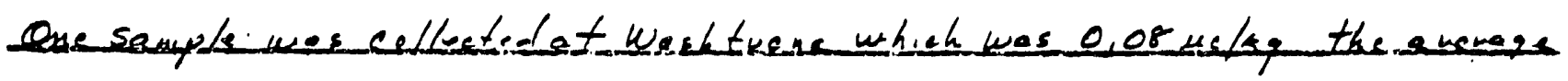
of all samples wes s0.04 uctuss

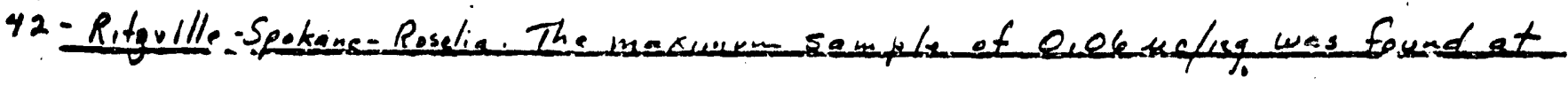

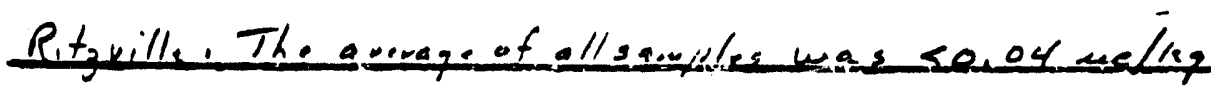

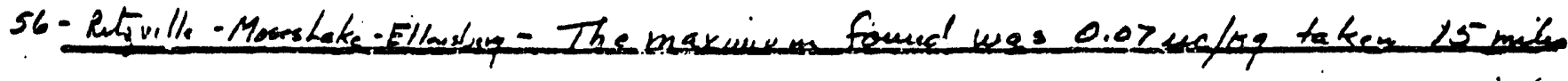
north of Lid. Theaverezen efoll somples was $<0.04$ ucky

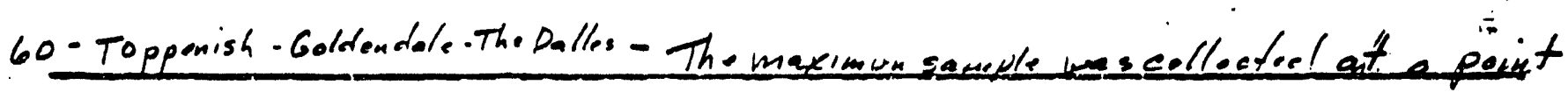
$1 ;$

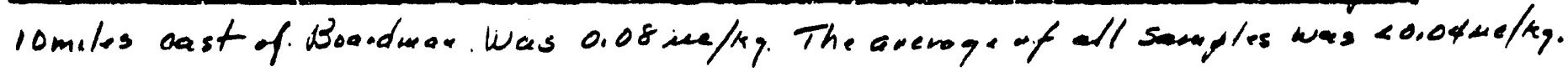

\section{Ground Conteminst102 Loagirsd with Portablo G. M. Listar -}

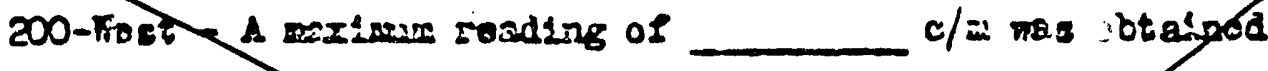

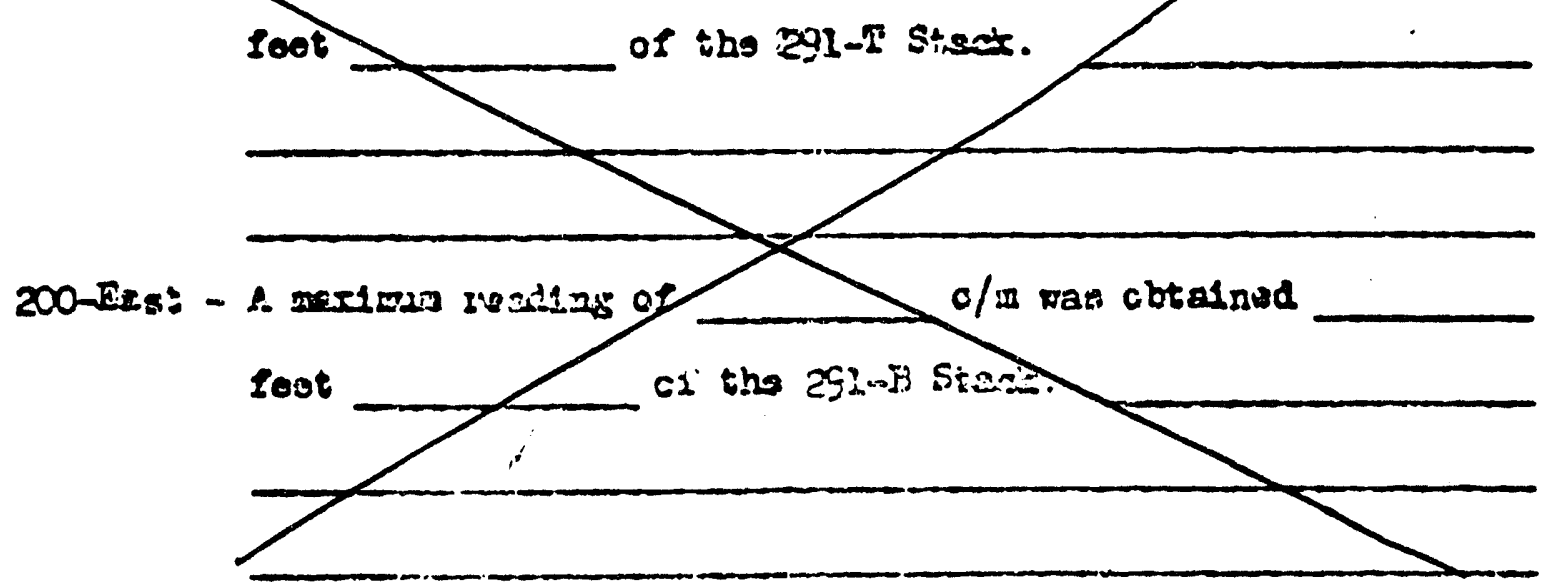

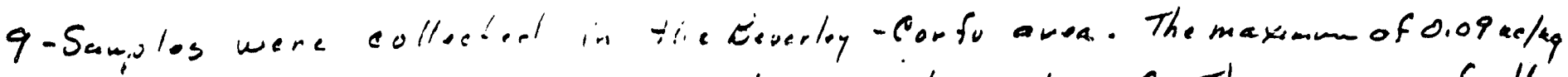
was found 2 miles north of Eltopia and $0.1 \mathrm{ruc} / \mathrm{kg}$ a + Corfu. The average of all samples was $0.05 \mathrm{mc} / \mathrm{k}$ ? 


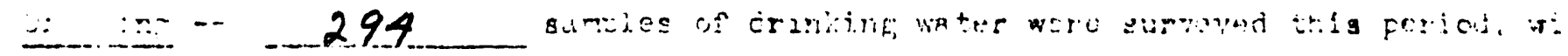

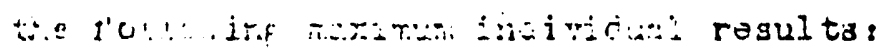

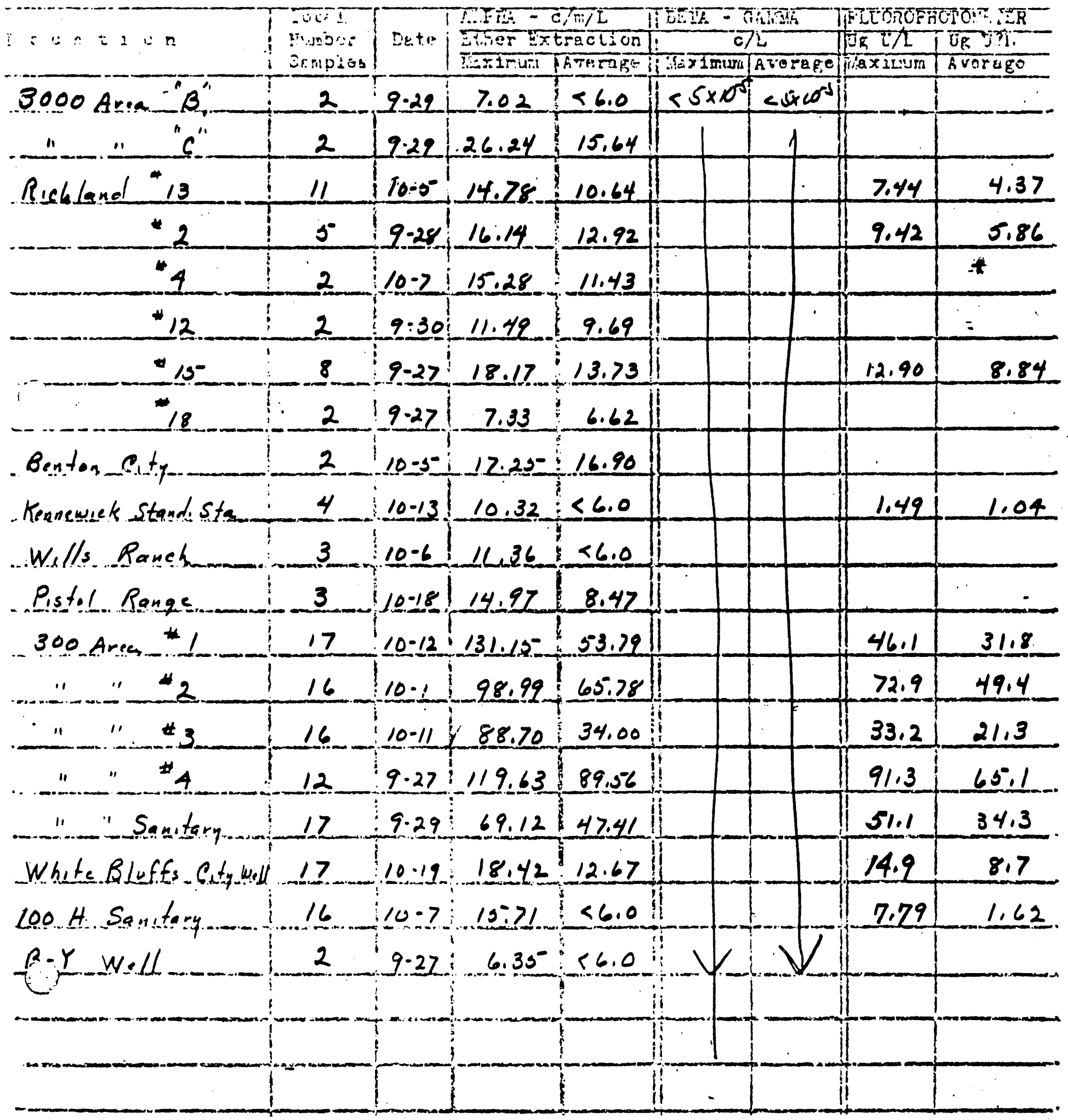


$\dot{1}$.

Five-A

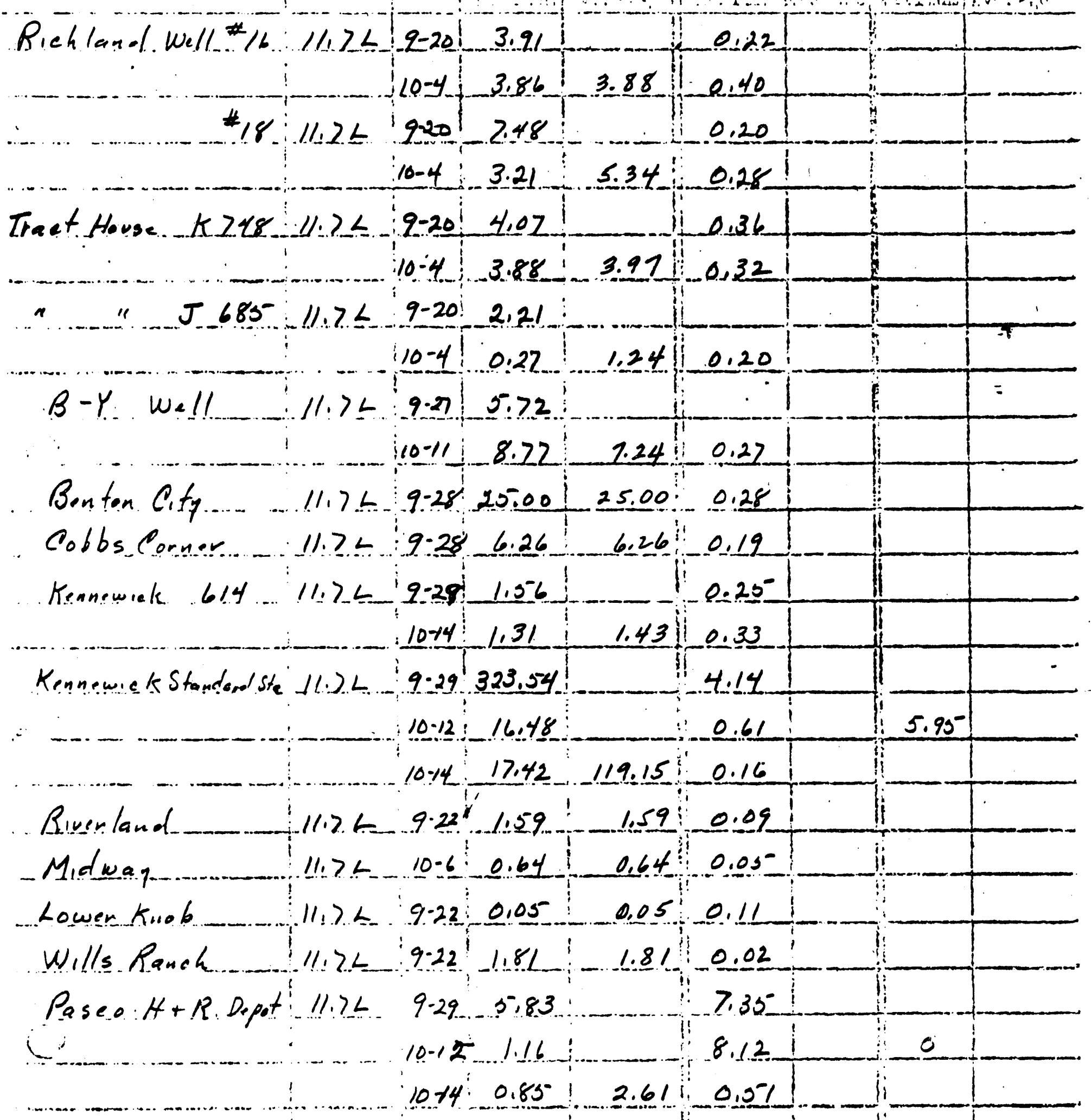

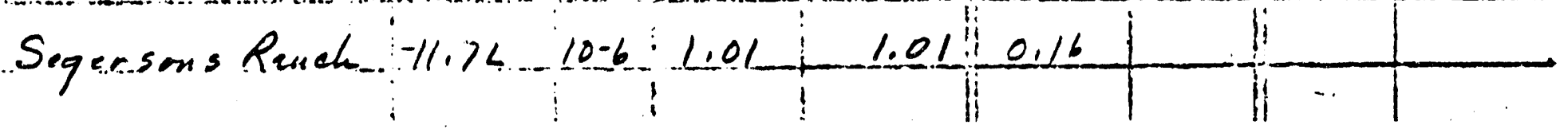




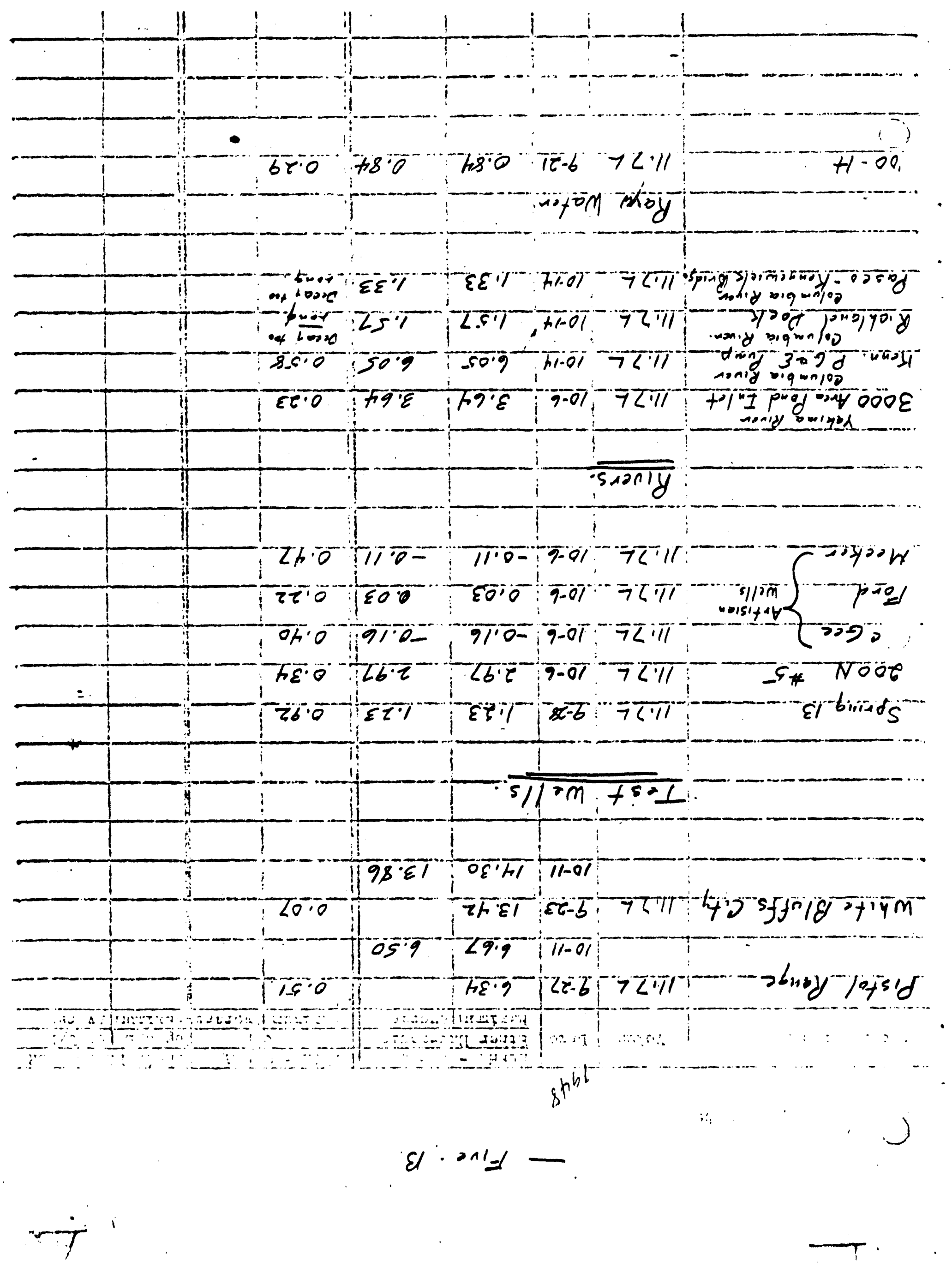




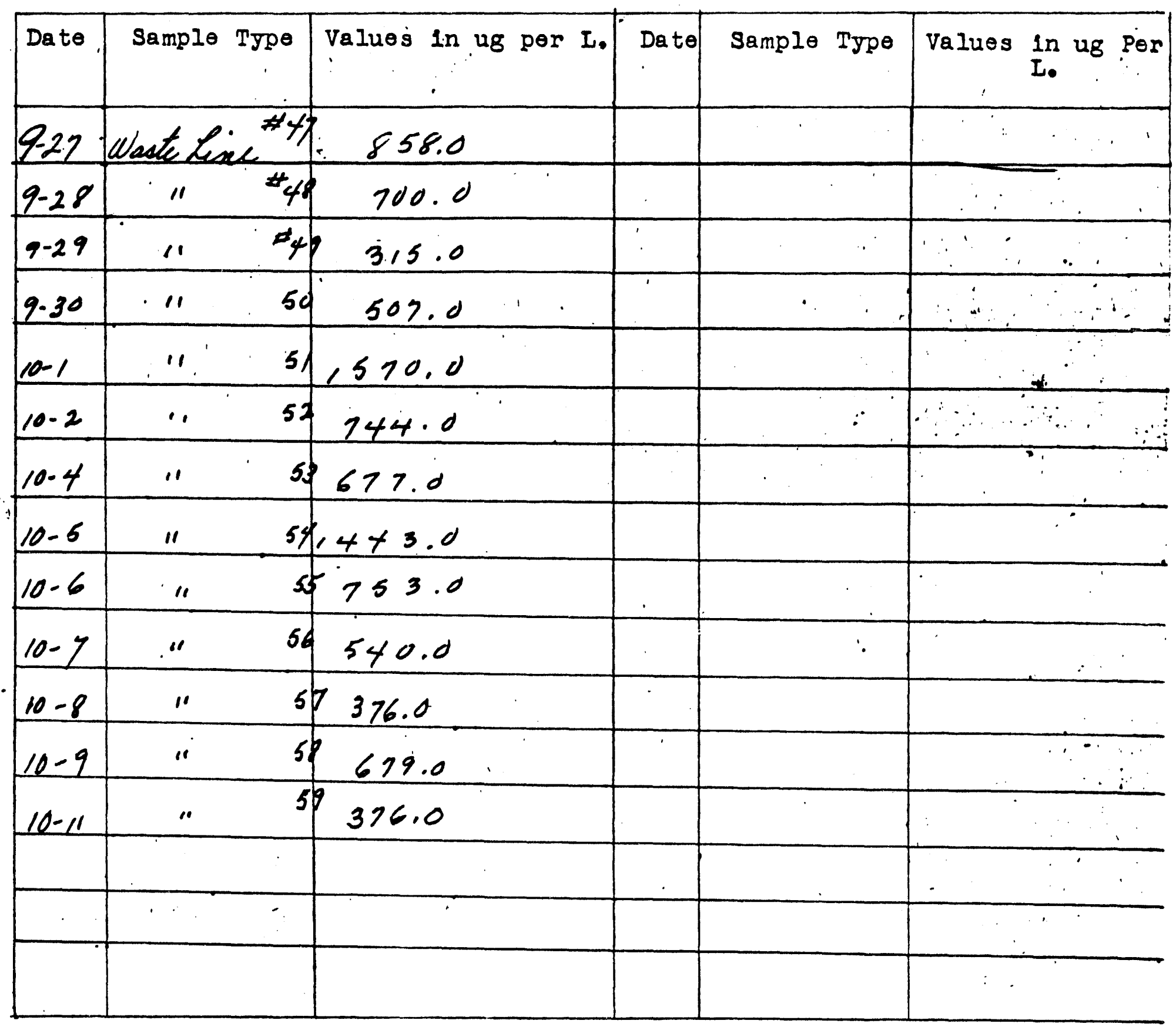


HATER (COMTinTHR) -

Toot risls -

12 -

There was no evirlence of Beta Lamma or Alpha cantemuation found.

\section{Bures -}

Colnowols Rgter -

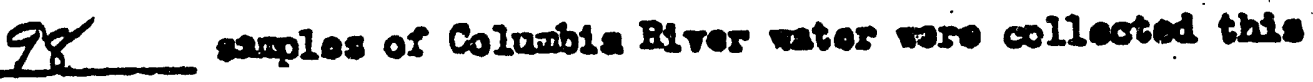
portod and aurvared, wth the folloxing realte:

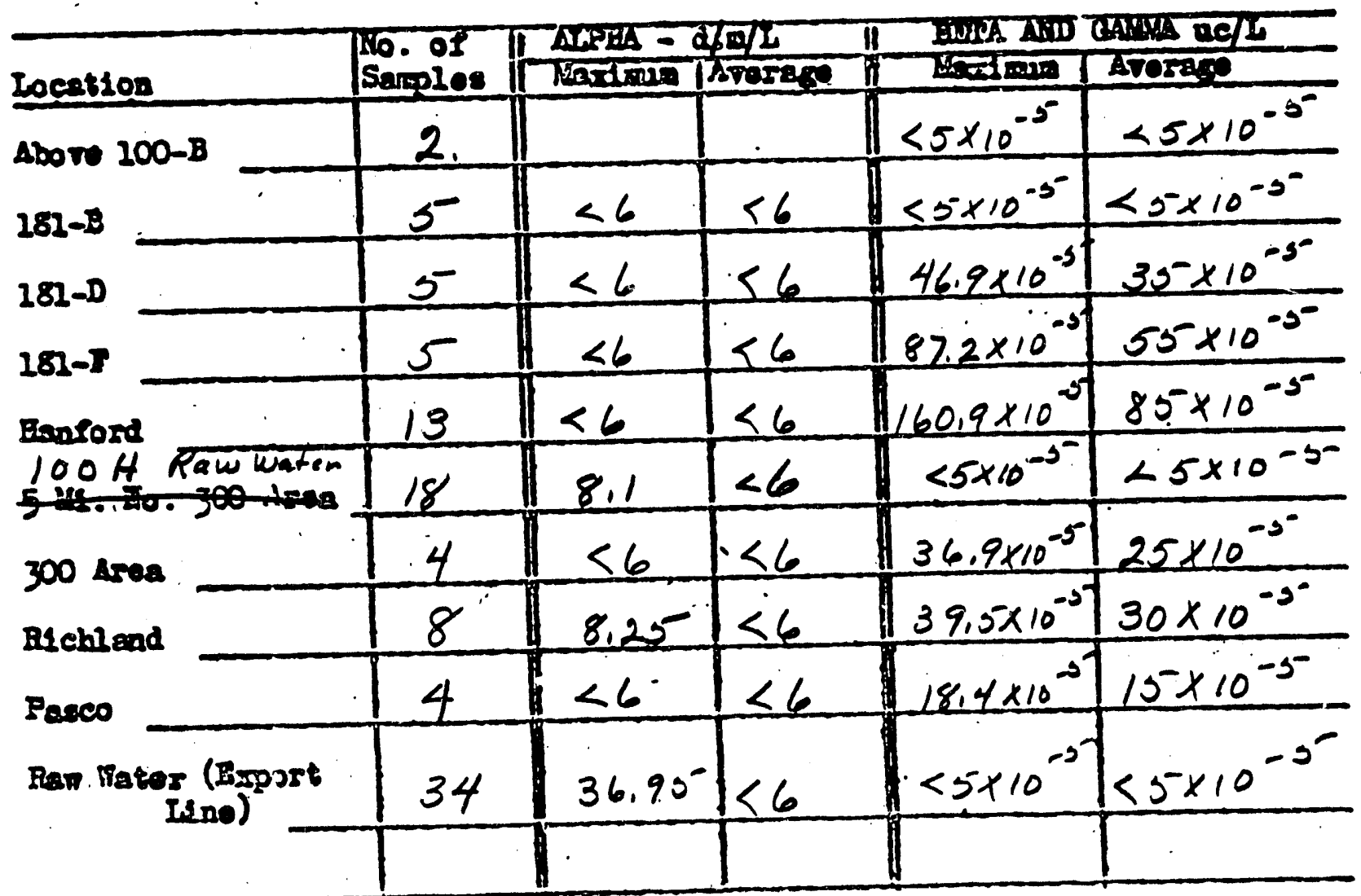



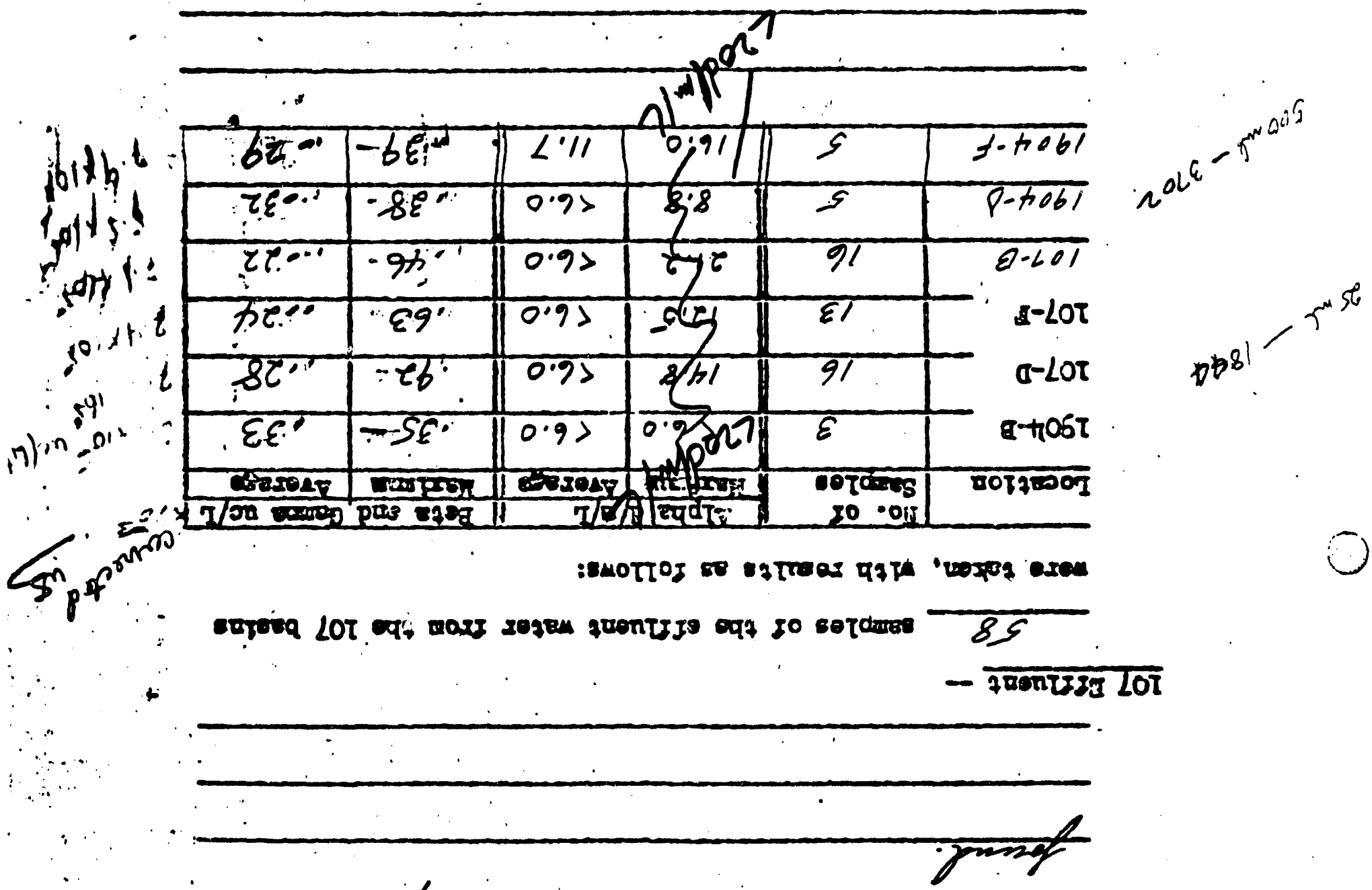

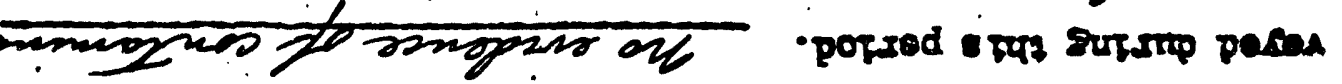

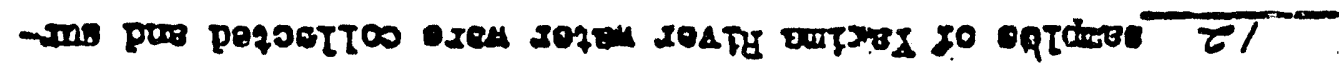

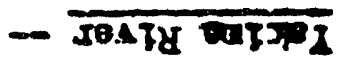

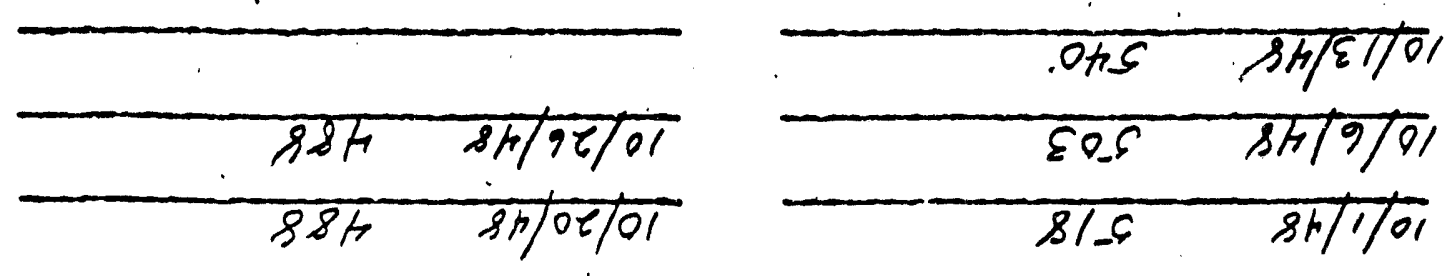

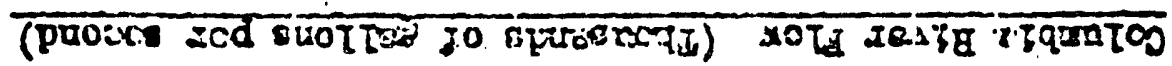

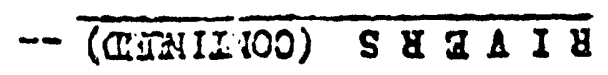


Fego Bigat

OASTE COITALINATIOH -

200 Host - Masto Solutiong --

\begin{tabular}{|c|c|c|}
\hline Iocation & Maxd & Hota knd Cen ued \\
\hline NTW Sng Inlot & $24 \cdot 33$ & $10 \times 10^{-5}$ \\
\hline Foet Sida & 36.04 & $10 \times 10^{-5}$ \\
\hline Sorth Sido & 73.30 & $10 \times 10^{-5}$ \\
\hline W" sxmp - Dot SLdo & 5.7 .60 & $<5 \times 10^{-5}$ \\
\hline$\therefore \operatorname{Ini} t$ & 24.02 & $6 \times 10^{-5} \therefore$. \\
\hline Ionodry DLteh - Inlot & 89.94 & $15 \times 10^{-5}: \cdots$ \\
\hline $600^{\prime}$ & 147.53 & $20 \times 10^{-5} \cdots$ \\
\hline 37. Drtan - Insot & 34,50 & $<5 \times 10^{-5}$ \\
\hline Itt. Onderpaes & 40.66 & $15 \times 10^{-3^{-}}$ \\
\hline
\end{tabular}

200 loot - Magte Sol1da -

Iocat10n

nII STam - S. SL do

$v^{2}$

(in

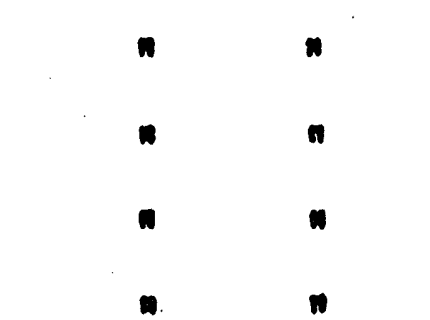

NT" Smanp - V. S1do
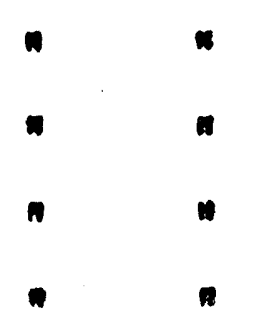

$200 w$. Petertion Rasin $(T)$

$200 E$ Retention Basen (B)

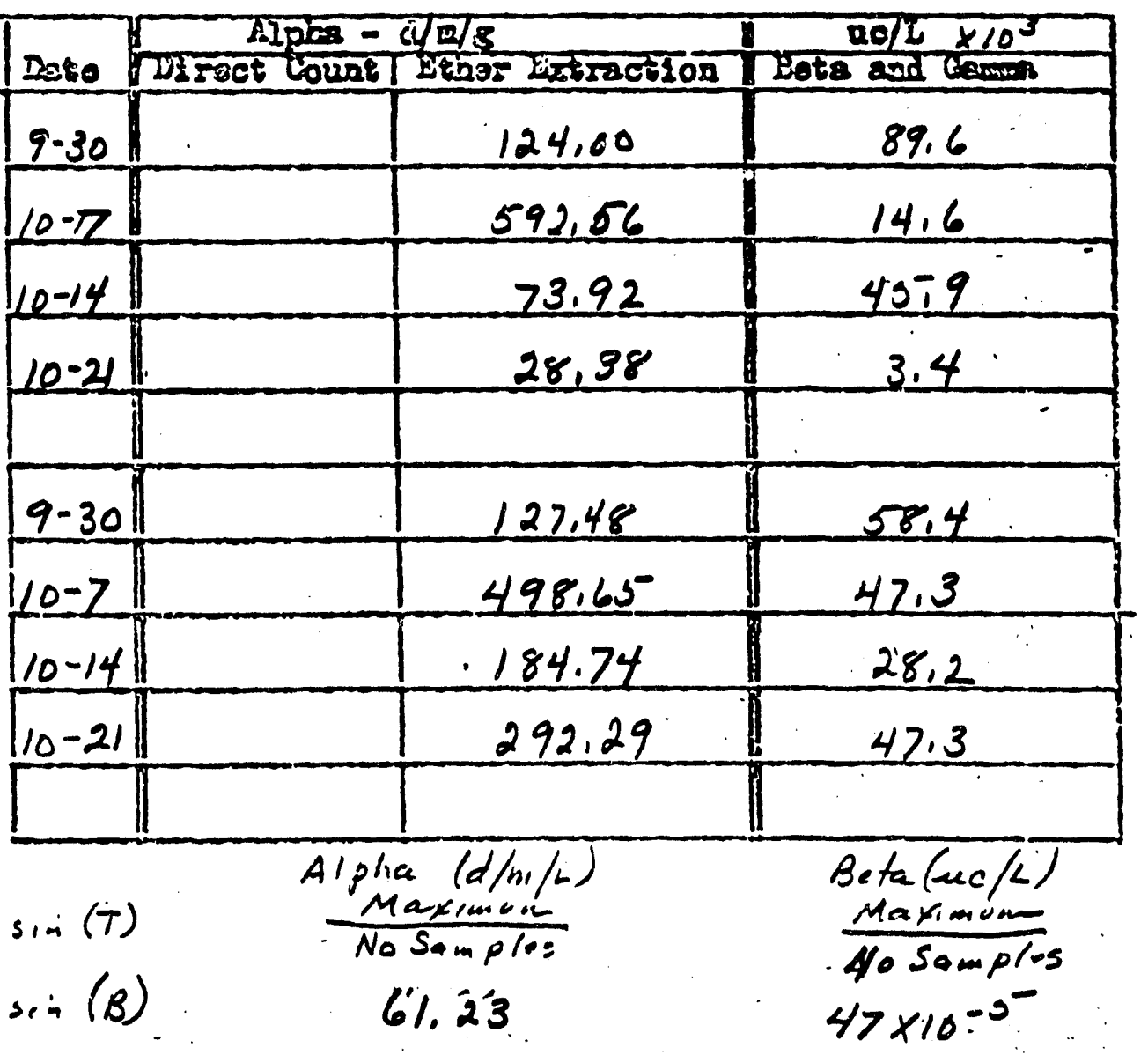




\section{Pago Mine}

$\because$

TASTE CORTAMINATIOS (CONTINED) 200 Foet - Maote Solida (Contioued) --

\begin{tabular}{|c|c|c|c|c|c|}
\hline \multirow{2}{*}{\multicolumn{2}{|c|}{ Iocation }} & \multirow[b]{2}{*}{ Date } & \multicolumn{2}{|c|}{ AIph -9156} & \multirow{2}{*}{ Bota $\cos / 4 \times 1$} \\
\hline & & & Direct Count & Bither sutract10n & \\
\hline \multicolumn{2}{|c|}{ Inoundry Iint } & $9-30$ & & 1766 & 539 \\
\hline 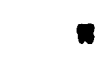 & $n$ & $10-7$ & & 32 & 43 \\
\hline$\omega$ & $\bullet$ & $10-14$ & & 552 & 97 \\
\hline$\bullet$ & $\omega$ & $10-21$ & $\cdot$ & 170 & 26 \\
\hline$\omega$ & 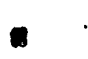 & . & . & & \\
\hline
\end{tabular}

200-Fot - Portable Q. M. Surver -

The andmin roading obtalned in this aras mas 200 c/a on mud at T-Sunap west side

200 Dast - Portable G. M. Survy -

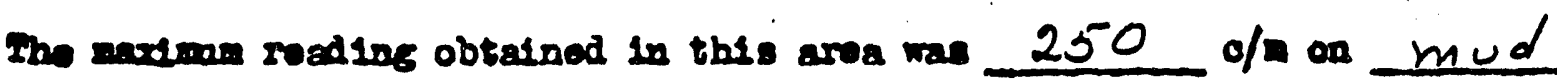
at 1000 ' from inlet of "B". diteh 
TASTE COATALINATIOR (COMTUED) -

300 Arag - Raste Solutions -

\begin{tabular}{|c|c|c|c|c|}
\hline \multirow[b]{2}{*}{ Bo. of Samples } & \multicolumn{2}{|c|}{ 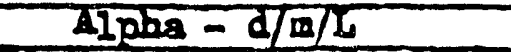 } & \multicolumn{2}{|c|}{ Bota and Gane - ac $/ 24 \times 10$} \\
\hline & Tandmen & Sroreg & Magna & Srerage \\
\hline 3 (IN/et) & 12659,3 & 7486.9 & 335 & 130 \\
\hline 3 (NW Corver) & 1389.0 & 1385.4 & 28 & 21 \\
\hline
\end{tabular}

300 Area - Waote Sol1de -

\begin{tabular}{|c|c|c|c|c|c|c|c|}
\hline Ios & 100 & & & Date & $\begin{array}{l}\text { Alphe } \\
\text { Ireet Count }\end{array}$ & 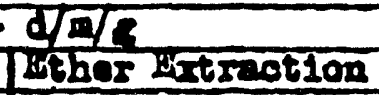 & II ne/zes $\times 10$ \\
\hline 300 & rea. & Fond & Inlet & $9-29$ & & 69 & 13 \\
\hline in & 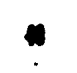 & 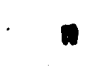 & $\bullet$ & $10-6$ & & 10 & 9 \\
\hline n & & . & $\varphi$ & $10-20$ & & 150 & 17 \\
\hline ๓ & - & $\bullet$ & $\omega$ & & & & . \\
\hline & n & $\bullet$ & n & & & . & \\
\hline$n$ & $\bullet$ & $n$ & in Cor. & $9-29$ & & 33 & 4 \\
\hline$\bullet$ & $\omega$ & ๙ & $\bullet$ & $10-61$ & & 6 & 14 \\
\hline 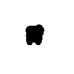 & $\boldsymbol{n}$ & - & $\boldsymbol{n}$ & $10-2 d$ & & 19 & 17 \\
\hline & $\bullet$ & - & 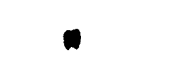 & & & & $\therefore$ \\
\hline n & $n$ & 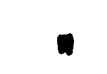 & - & & & & \\
\hline
\end{tabular}

200 Area - Portable Q. 4. Sorver -

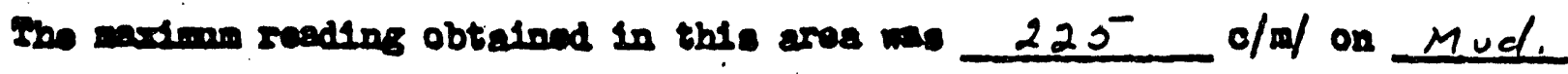
at $\ln l e t$ to 300 Area Pond.

\section{Morth Area Fasto Survers -}

Iocation

IIn Drteh

mpa

ma
Yax1:un

P.ad 1 a.

\begin{tabular}{|c|c|c|c|c|}
\hline$<0.05$ & $\mathrm{Krop}_{\mathrm{p}} / \mathrm{H}$ & $<0.0 \mathrm{~s}-$ & $\mathrm{NOR} / \mathrm{KR}$ & 900 \\
\hline$-5=0$ & $n$ & -215 & $n$ & 44,000 \\
\hline $4.5^{-}$ & if & 2.0 & $n$ & 32,000 \\
\hline
\end{tabular}


Pow Devan

(i) AIR HOEIIORIXO

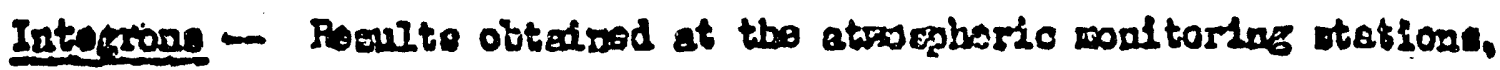
as cosparad to tho provlous wonthe aro as follows: (Mrenp/24 hours)

Iocation

$200-3$

200-D

100.9

$200-\pi$

2000.

* Paresand

Inimord

300 srea

700 asea

remondet

Panos

Bater Csty
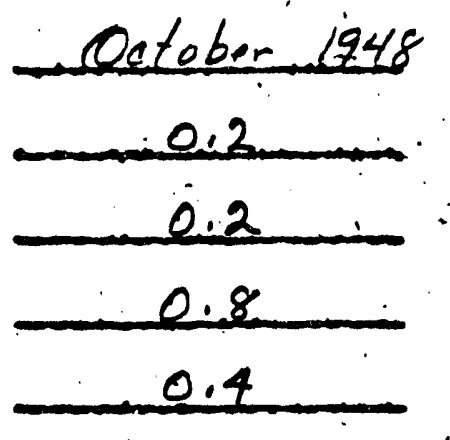

Ois

0.6

$\ln 3$

1.5

$<0.1$

soil :

0.2

$0,5:$
September 1948 0.7

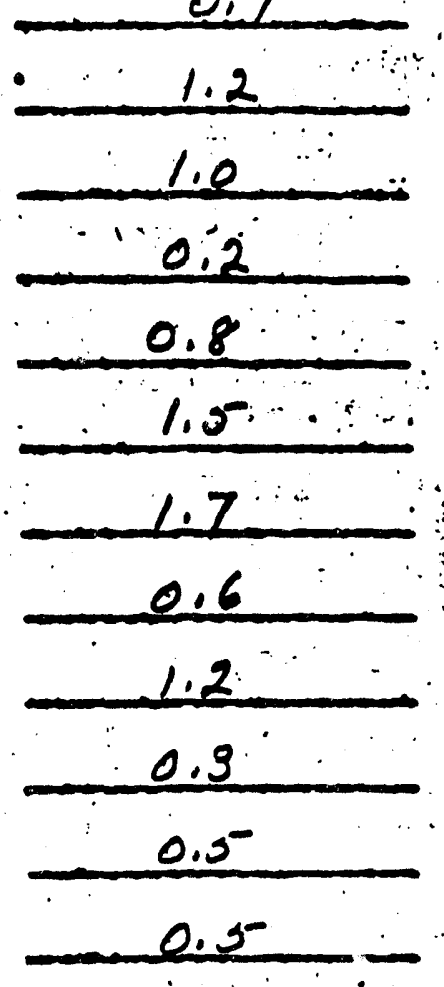

* New. Instrument enstalled. 


\section{Pase trolve}

(E) $\triangle$ IR NONITOBINO (CONTINUED) HCN Chambers - A wrvey of the "CN Chambers rogults for the sonth of October 1948. as compared to tho provious wonth, are as follcre: Dosege in virepl 24 hrs.

Iocations
$100-B$
$100-D$
$100-F$
$200-1$
$200-1$
300 Aran

\begin{tabular}{|c|c|}
\hline Oetober 1948 & September 1948 \\
\hline 0.3 & 0.3 \\
\hline 0.4 & 0.3 \\
\hline 0.4 & 0.4 \\
\hline 0.3 & 0.3 \\
\hline 0.5 & 0.6 \\
\hline 0.5 & 0.4 \\
\hline
\end{tabular}

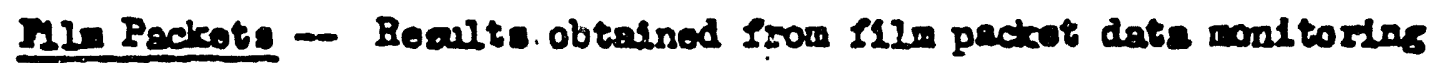
chor the radiation Iovele to be $\leq 5.0$. mrep 24 hre. at all locatione. Tronty thres peckets wers in eorrice. No significent reading. 


\section{Pag Intsen}

A I R NOEITORIRG (CONTINIE) -

Detachable Chambers - Arorago dosage rocilings obtalned with dotachablo chambers, as comparod to tho proviou nonth's observation, are as follows: (all readings are averago mirep $/ 24$ hrs.)

Iocations

100 Aroas and Bavirona -

Route 14108

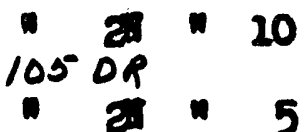

White Bluffs

- 11-4" 1

Houfond 614 6/d,

- 1 and Boato 4 Karth

Hanford 101 sid,

Ithen 5 nies $200-$ -

Ronte 45 usie 6

- Iน".6

- $3: 1$

$234-235$ Area-200 west.

Intcorolog - 200'.

$2415 x-200 \omega$

It thin 10 ales 200-2 -.

Boute is L4I0 10

- $10 \div 2$

- $20 \backsim 3$

- 25.4

Doar 300 Area -

Boute 4s ris 16

- $49 \cdot 22$

700 Area
Qctober 1948
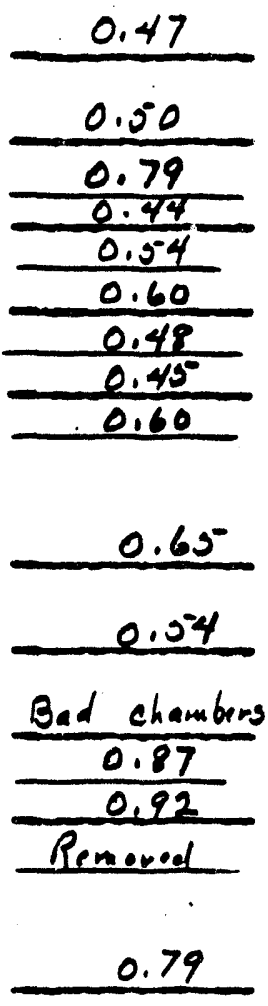

0.74

Bad chambers

0.86
September 1948

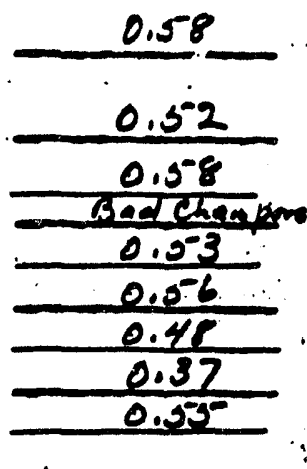

$0.5 y$

0.71

$0.69^{\circ}$

$\frac{\text { Not installed }}{\frac{0.60}{0.54}}$

0.70

0.60

Bad chembers.

0.82

\begin{tabular}{lll} 
Bentencity & 0.48 & 0.79 \\
Ruerland & 0.48 & 0.48 \\
\hline
\end{tabular}




\section{Pago Tolirticen}

\section{CIR YOHITORI HQ (CONINUED) -}

Constant Iodine Honitoring -- I' I concontrations os moatared by C. I. Units In the month of October $\frac{1948}{1}$ Maximum ros rollow:

Iocation.

200

$200 \times$ - Towor 4

Cable Nountaln

Blobland

200 west Gate
8 bour concontratione uch

Moan avrag concantrations for October $1948 \mathrm{nc} / \mathrm{L}$

$\frac{\frac{5.5 \times 10^{-9}}{5.5 \times 10^{-10}}}{\frac{8.7 \times 10^{-10}}{1.1 \times 10^{-10}}}$

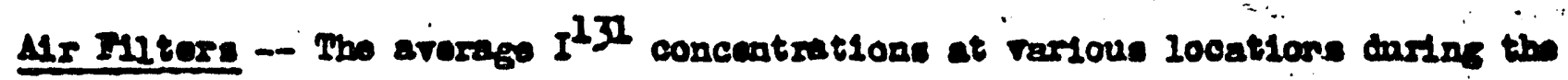
math of Oetobee 1948 ware as followa:

Iocation

Pasco

300 Area

200-5; Tower th

$100-D$

200-1 cate

Bantos C1t5

Banfond 614

Hanfard $101 \mathrm{R} / \mathrm{dg}$

White Bluffs

LOS-DR
Oeteber 1948

$\frac{1.6 \times 10^{-10}}{3.9 \times 10^{-10}}$

$3.5 \times 10^{-9}$

$\frac{3.5 \times 10^{-9}}{1.6 \times 10^{-10}}$

C. I.Unit Installed

i.

$1.7 \times 10^{-10}$

$\frac{2.6 \times 10^{-9}}{4.3 \times 10^{-10}}$

$1.7 \times 10^{-10}$

$2.9 \times 10^{-10}$
September 1948

$1.6 \times 10^{-1 / 0}$

$6.0 \times 10^{-10}$

$8.9 \times 10^{-9}$

$1.1 \times 10^{.10}$

$8.2 \times 10^{-10}$

$7.1 \times 10^{-11}$

$1.2 \times 10^{-9}$

$2.5^{-} \times 10^{-10}$

$9.3 \times 10^{-11}$

$1.3 \times 10^{-10}$ 
Page Mriten

$4 \times$

A I MORITORIEG (CONTINUED) -

Rain Sangiline -

$65^{-}$ rain esples wore collected in this porlod. Rosults obtalnod are surmarisod an fol 'ore:

\begin{tabular}{|c|c|c|}
\hline Location & Landman ac/L & Averese uc/L \\
\hline Within 200 Iroa & $8.5 \times 10^{-2}$ & $1.5 \times 10^{-2}$ \\
\hline$W$ then $200-\pi$ & $1.9 \times 10^{-2}$ & $2.6 \times 10^{-3}$ \\
\hline 100 Areas and Inritroas & $2.7 \times 10^{-4}$ & $1.2 \times 10^{-4}$ \\
\hline Intormediate on Aren & $3.6 \times 10^{-3}$ & $8.1 \times 10^{-4}$ \\
\hline Outbifos - ort Area & $8.8 \times 10^{-3}$ & $1.0 \times 10^{-3}$ \\
\hline
\end{tabular}

The maxemum eff area semple wes collected at Ruealand on $9 / 22 / 48$ 


$$
H w-89074 . \quad \text { cic. } 12764
$$

BEST COPY AVAILABLE

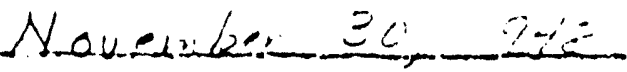

:0. W. Suggl:y-1

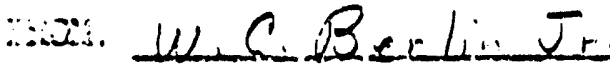

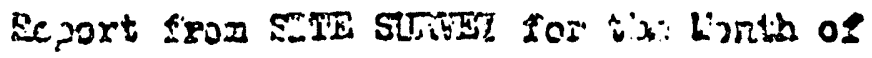

Nevember 1248

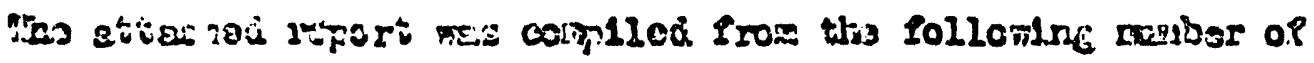

:72.5 30:

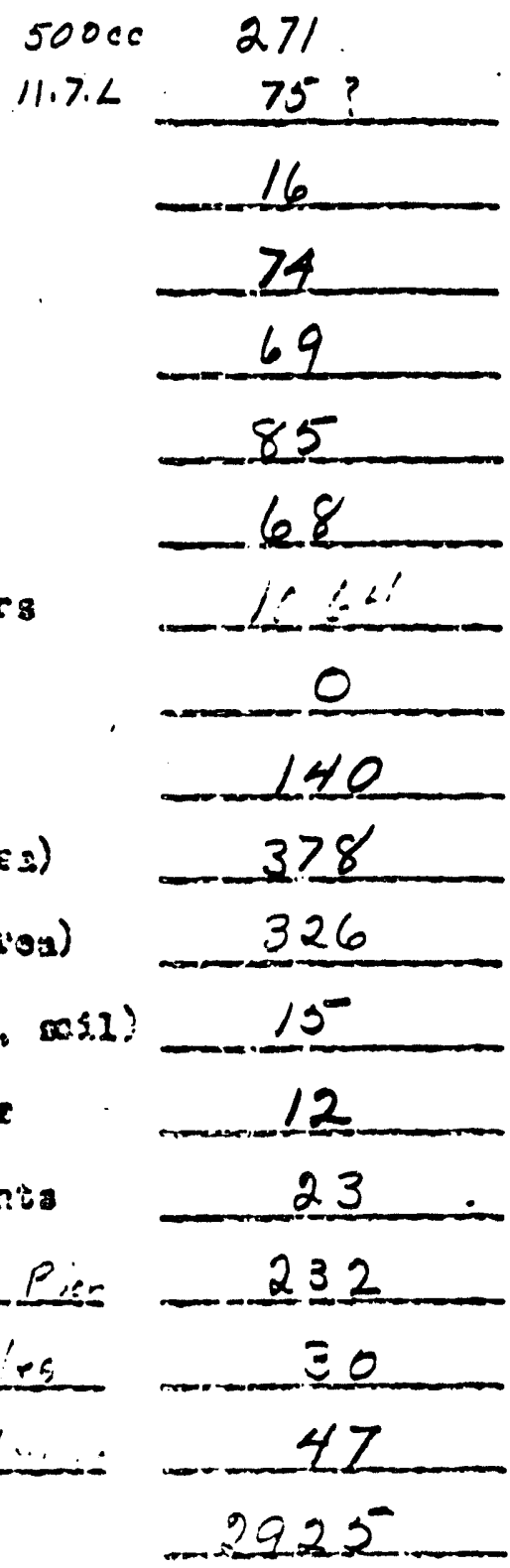




\section{$\operatorname{lag} \rightarrow \operatorname{Tas}$}

BNMn:TCA -

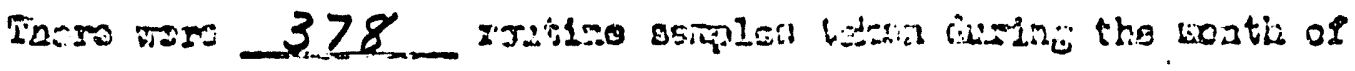
- The levels of centemustion os indicat..l

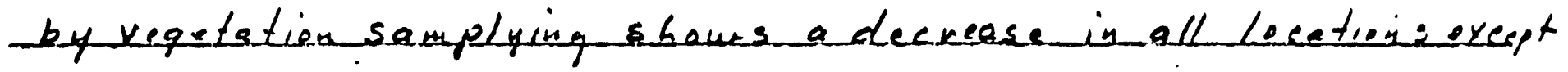
in the Houfecel acead. There were po highly signifisant xadugs. -on requtation during this geriad.

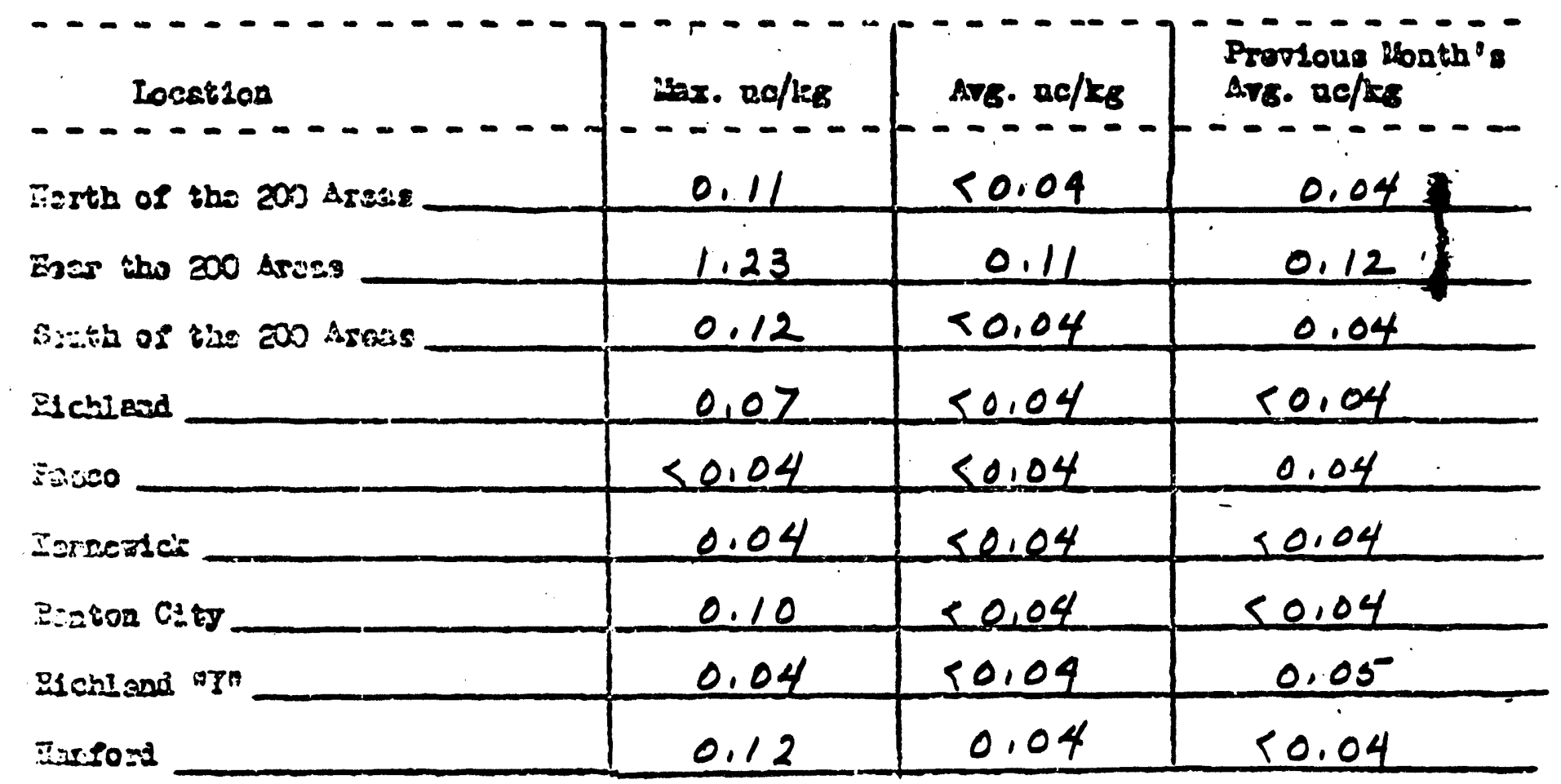

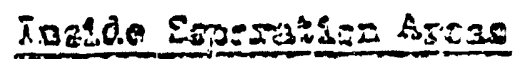

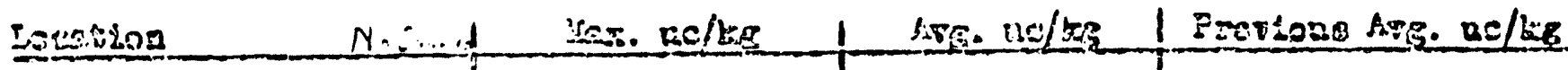

$200 \operatorname{lings}$ 20

$\underline{4}+1$ c. 10 0.09

¿OO INAs

13

C.teci, $20=0 \div 24$

1.20

6.30

0.24

0,12 0.32 


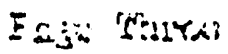

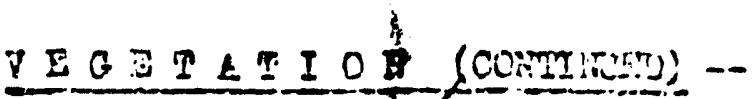

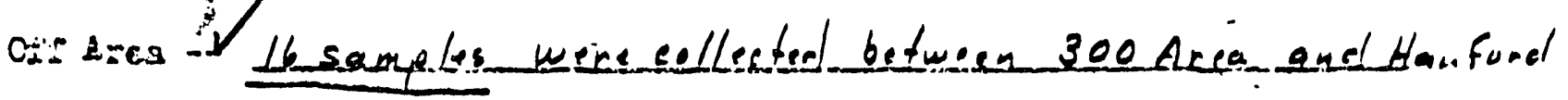

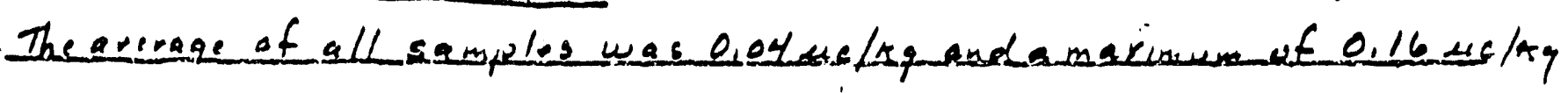
found 5 miles se of Hew ford

19 samples werc collected in the Goose EgqHill orra. Thearerape of

all samples wes 0.09 uelsg ind the maximumes 0.26 uelsp found directly south of 200 cest gater

V69 sample were colleoted one'survey of Benten Gap thanuenge

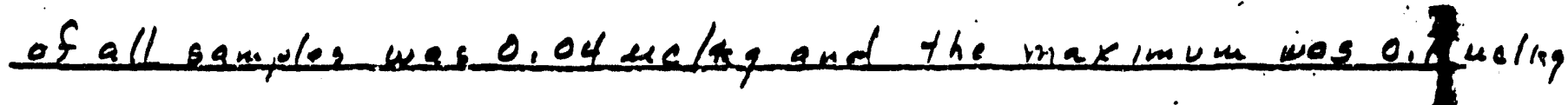
found at the 2600 feet levele

$$
\text { (eonfinued) }
$$

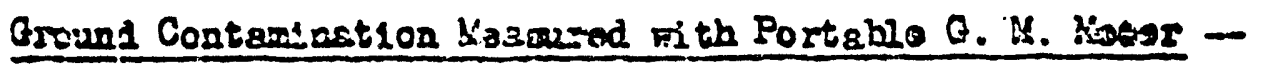
200-Trat - A maximum rasding of $7500 \mathrm{c} / \mathrm{a}$ wo obtalnod 1500 2 foot SE of the $291-\therefore$ stact.

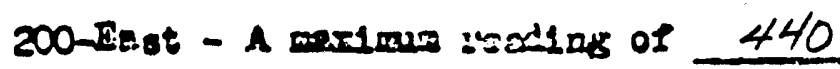
o/w nas obtalnod 1000

Poot Elest of the 291-B Stads. 


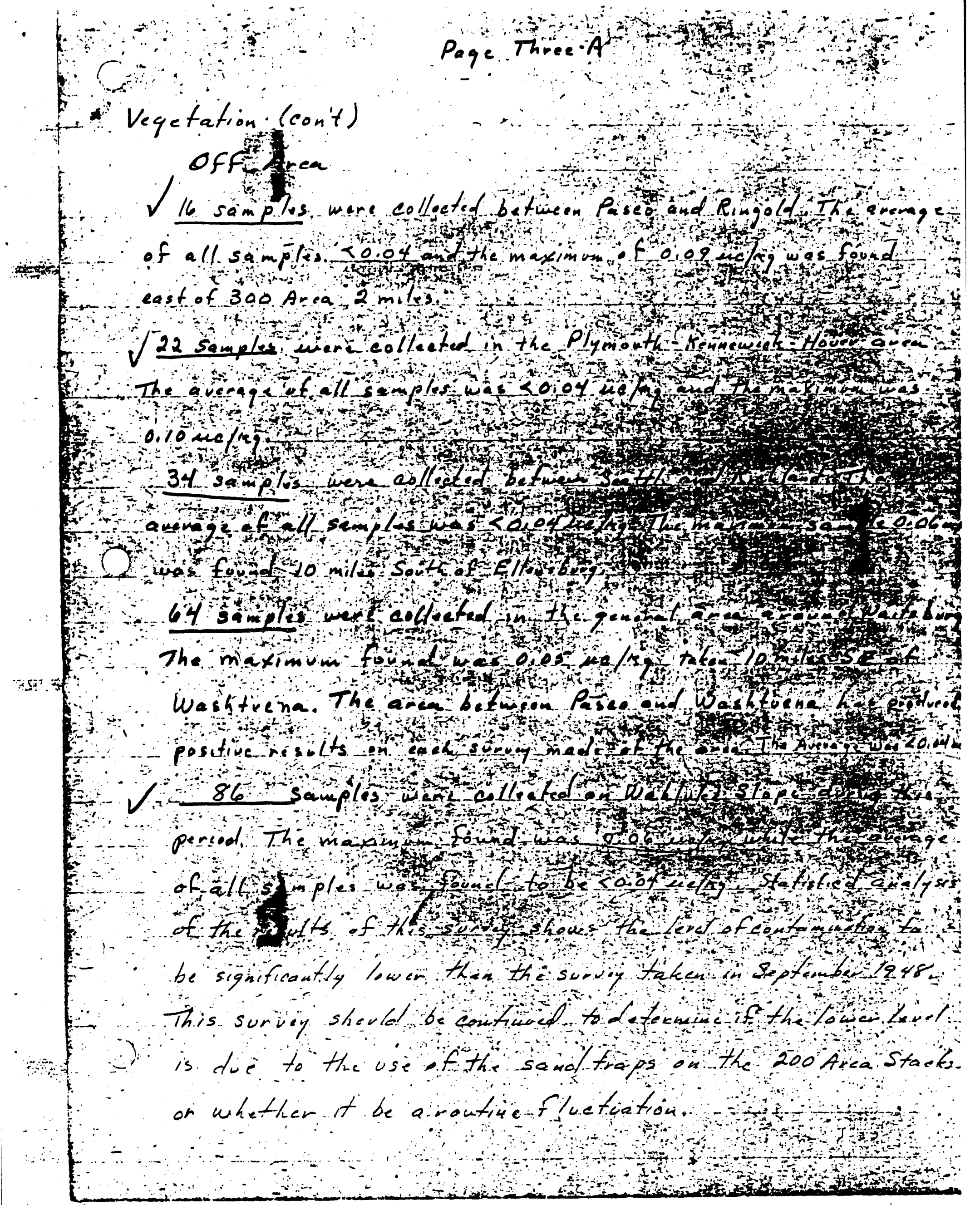


Nov. 30,1948

Columbia River Mud- 68 Samples of mud from the Columbia Riga

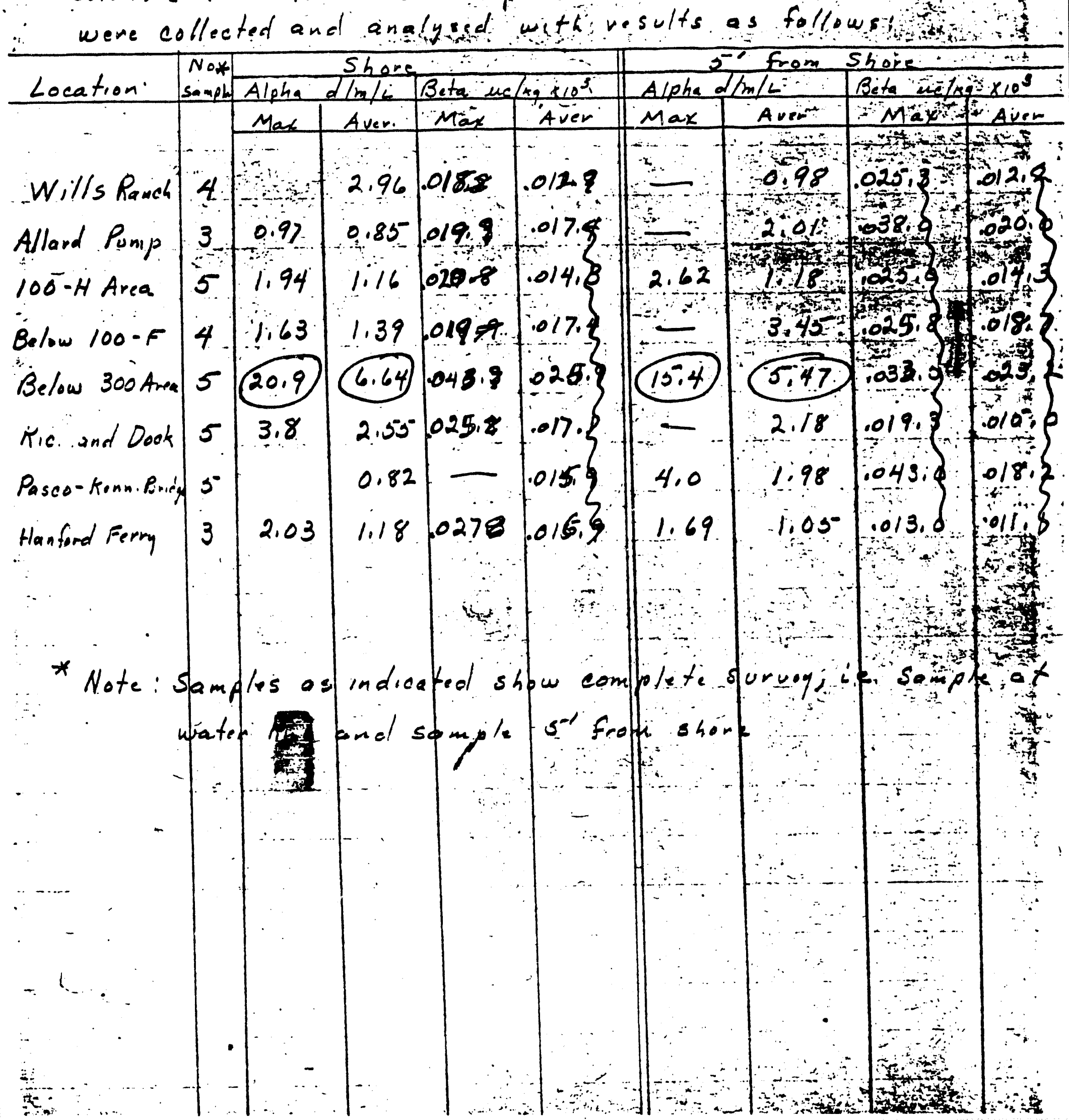


Propo FIV0-k

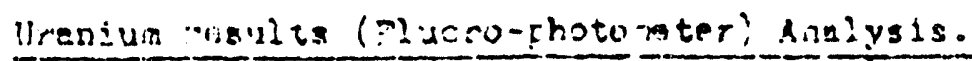

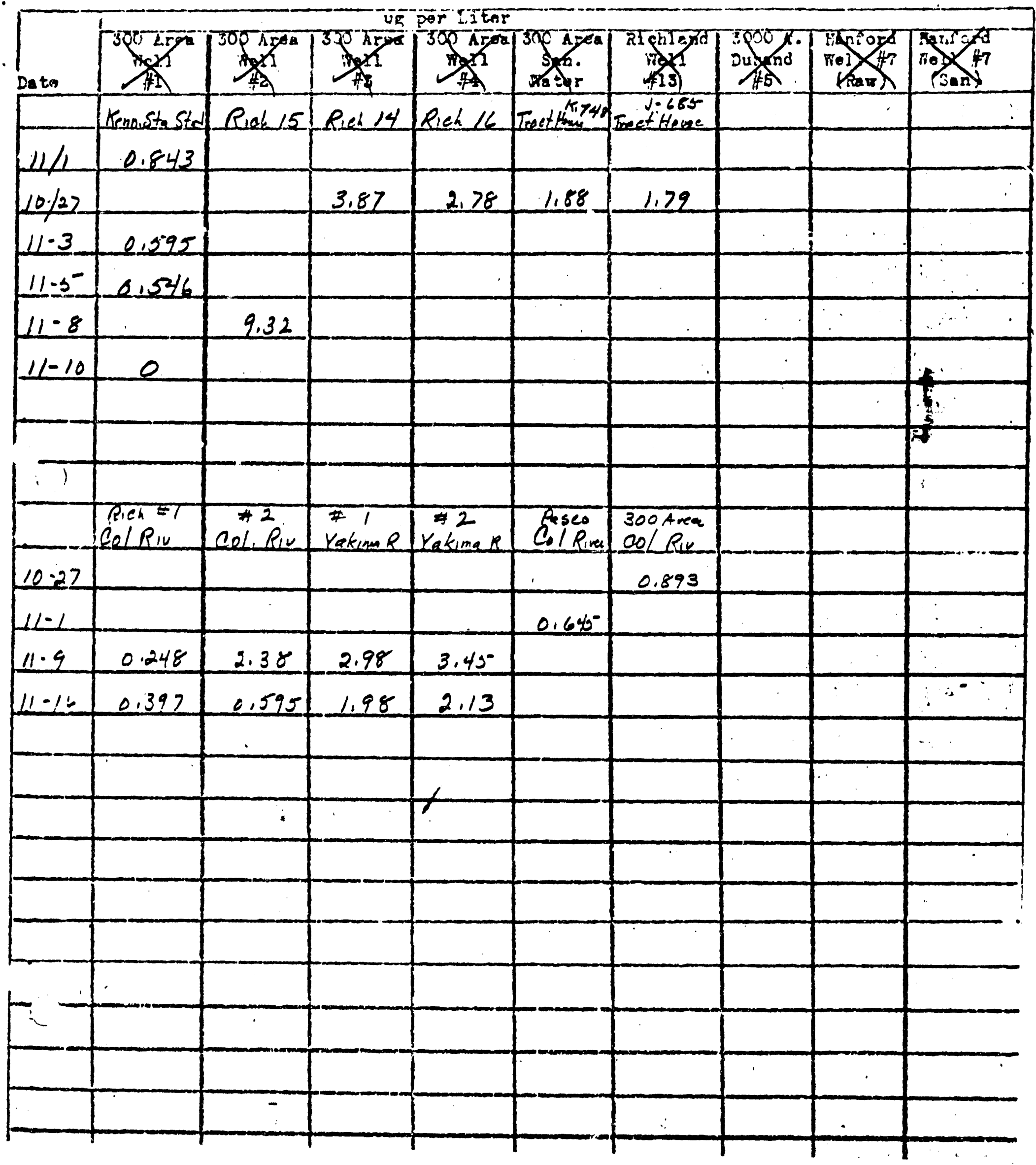


(1)

6

$\cdots \quad \ldots 271$

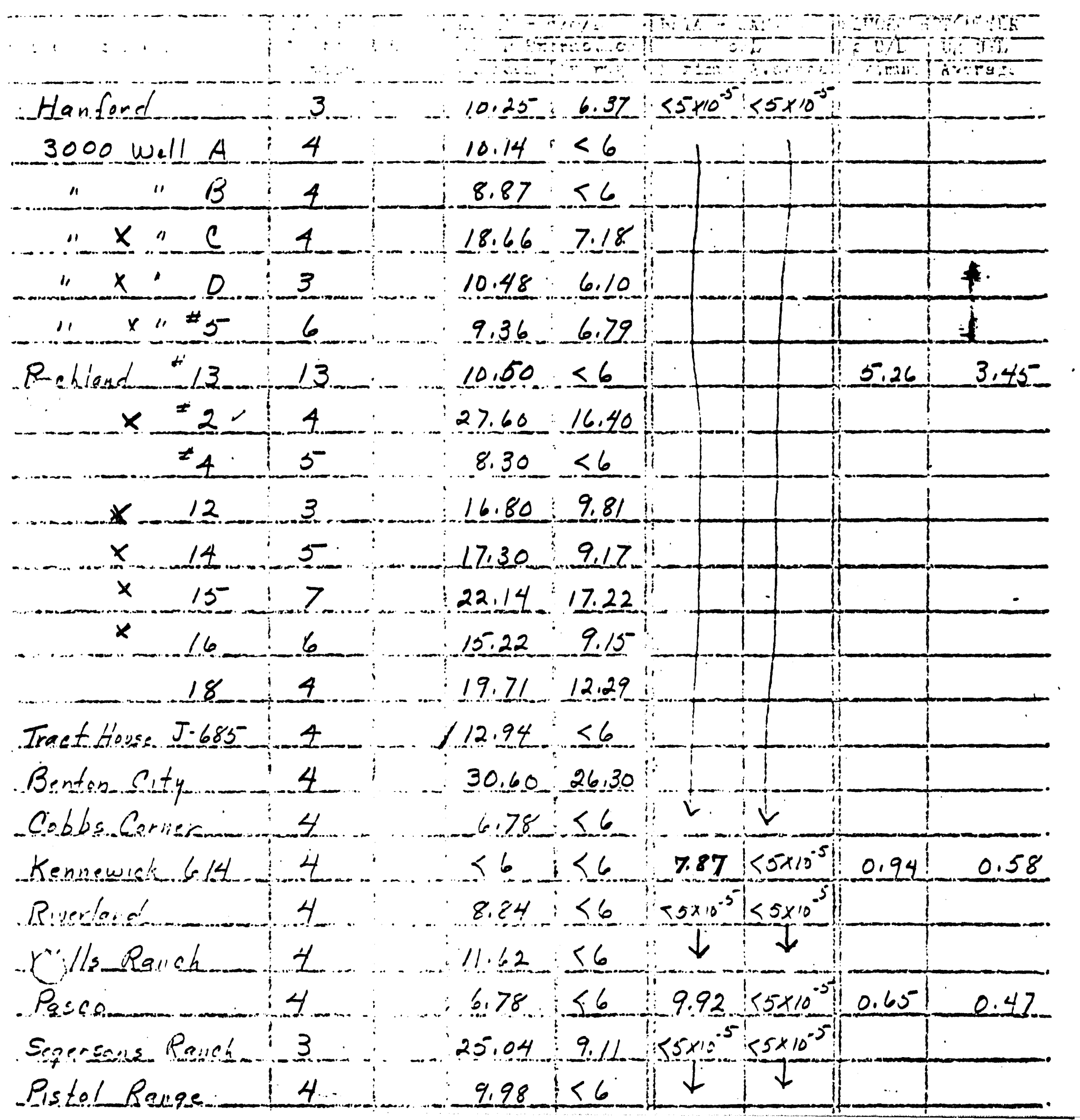


300 Area well $\ldots 1.21$
$2 \ldots$ $\begin{array}{ccc}\ldots \ldots & \ldots & 3 \\ \ldots & \ldots & 20\end{array}$ San 21

White 13 luffs $c_{1} ! y \quad 16$ 100-D Sanitary

4

200E

4

$B \cdot Y$
$96.053 .205 \times 10^{-5}<5 \times 10^{-5}$ 108.450 .90 $123,8 \quad 32.00$ $122.6 \cdot 80.20$ $94,3 \quad 39,62$ $21.2,1242$ $30.18 \quad 13.08$ $9,98<\leq 6$ $10.16 \div 6$

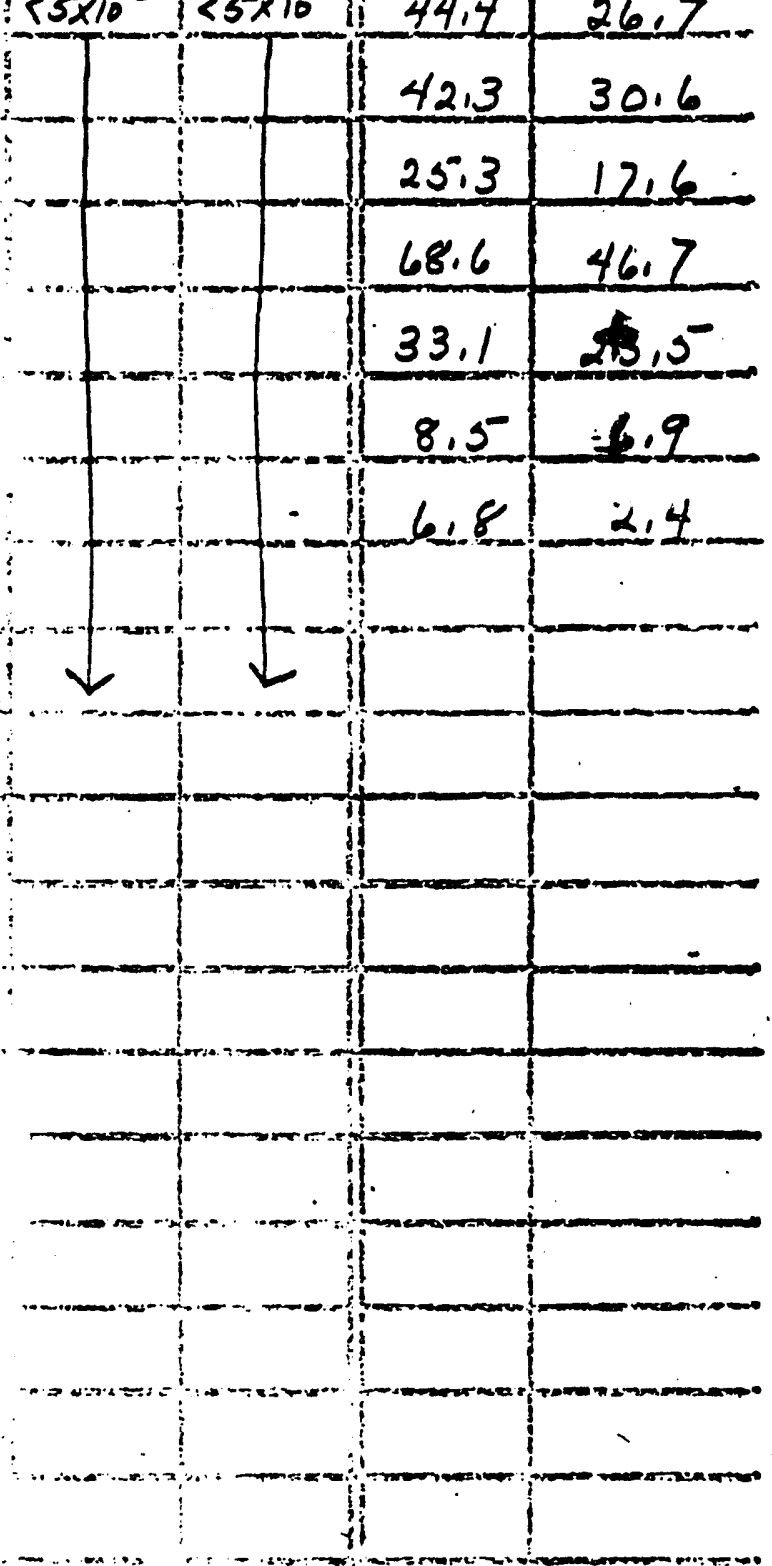




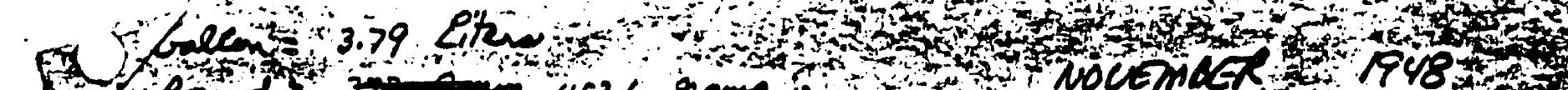

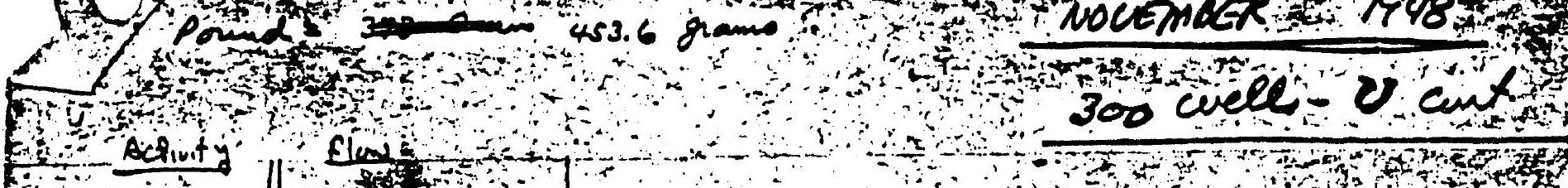

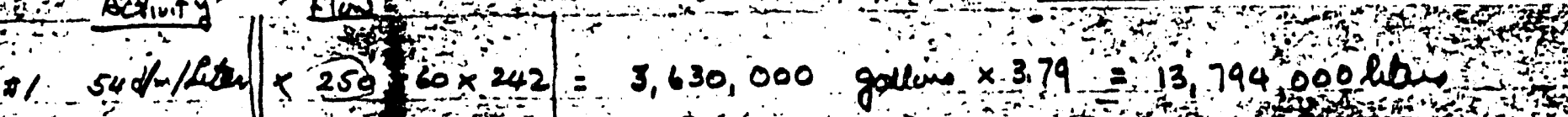
6.

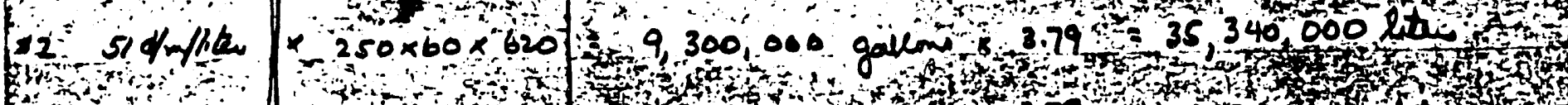

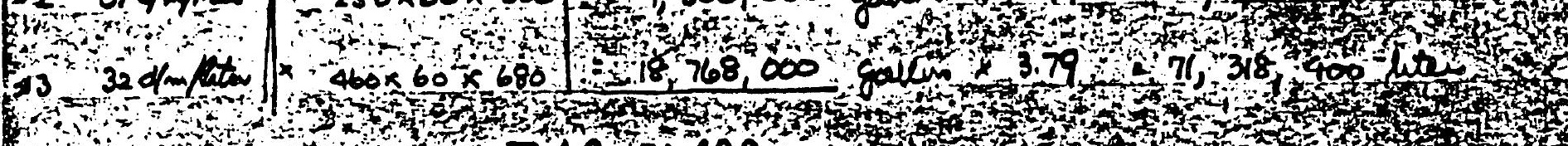

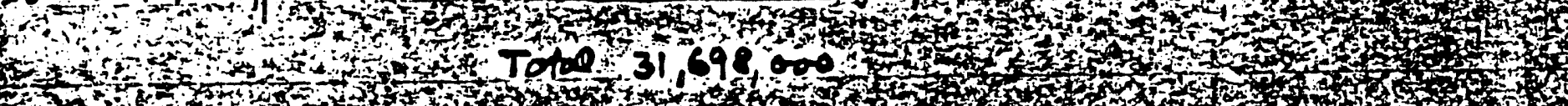

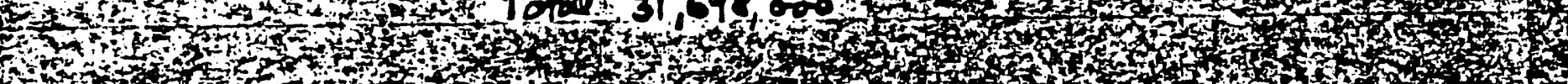
2. ry

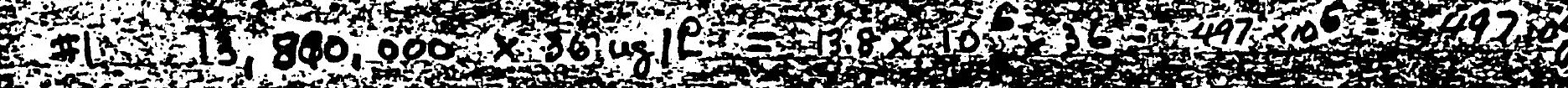
2.

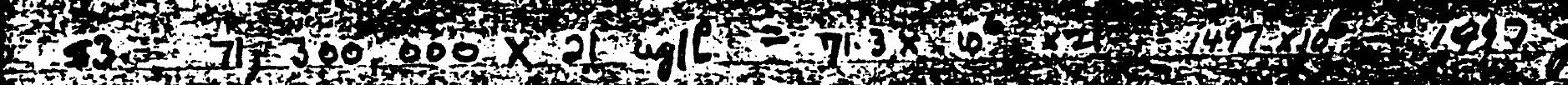

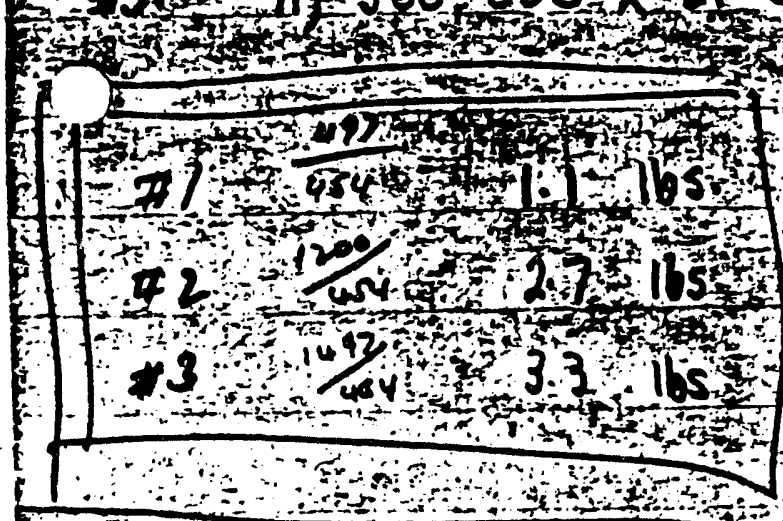

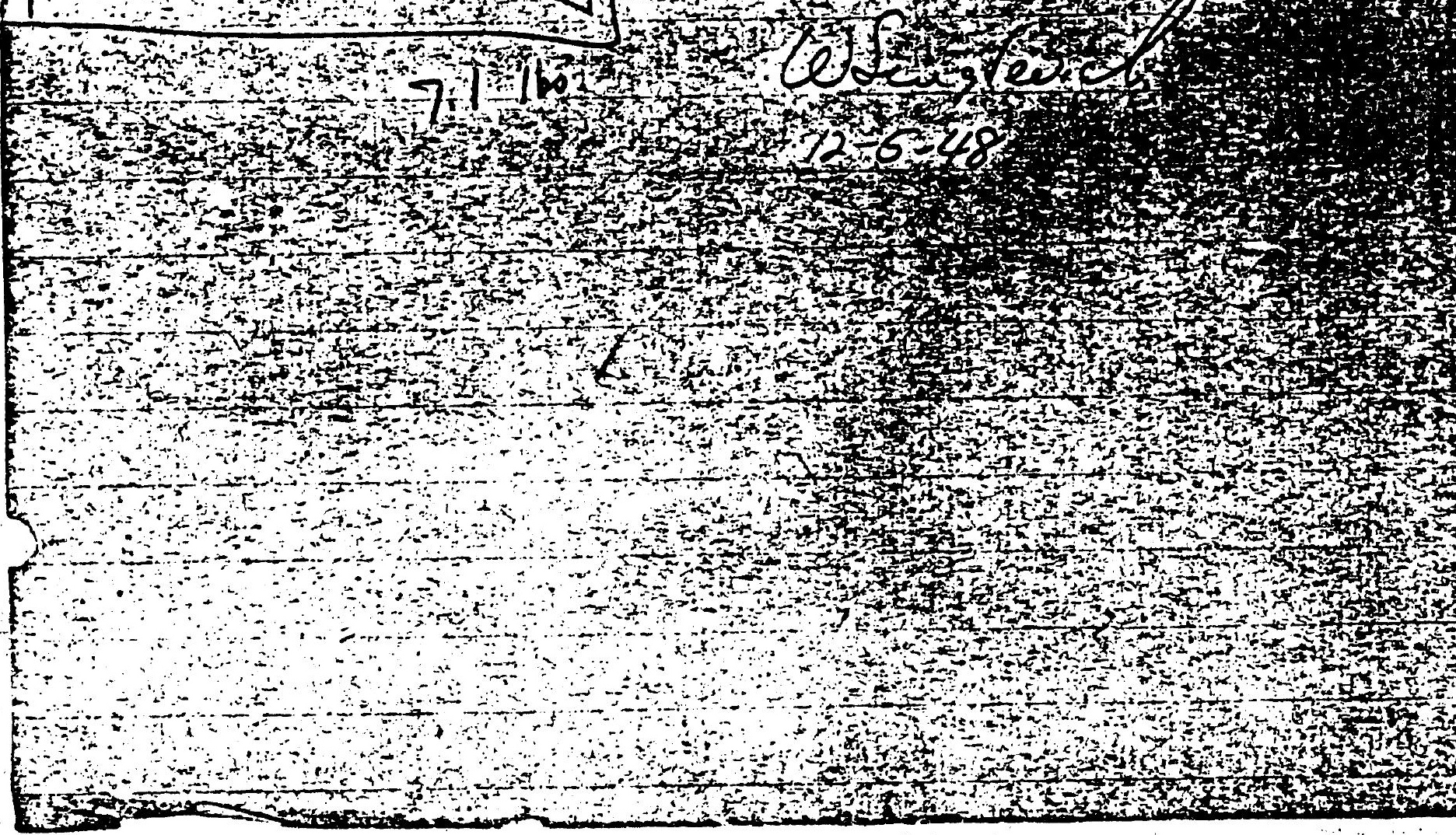


$\mathrm{c}$

$3, x+40$

Special Watir: Samples

North pend Washingtan.

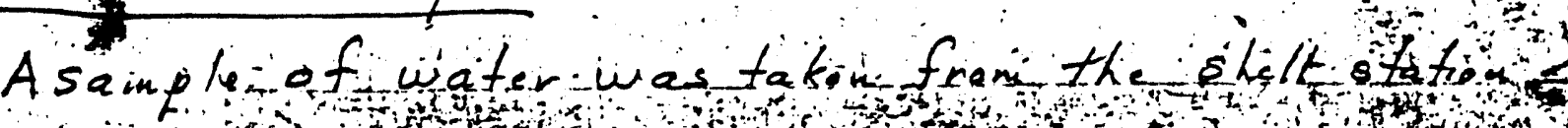

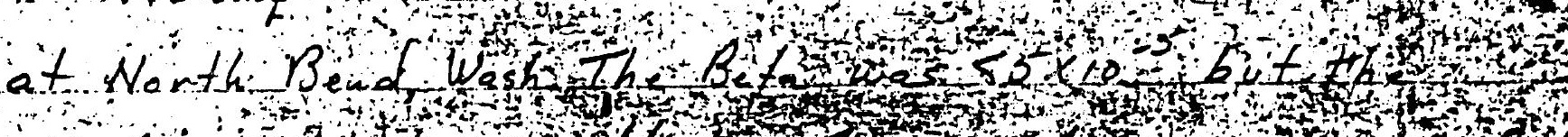

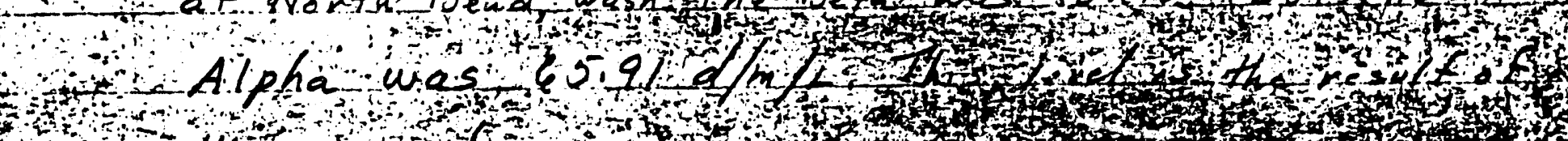

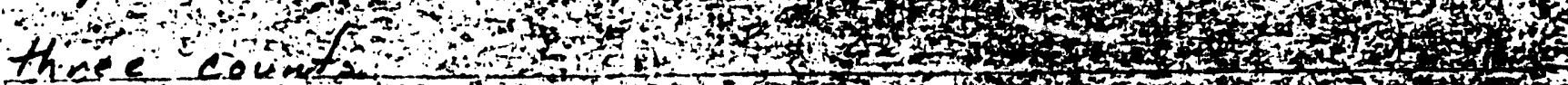

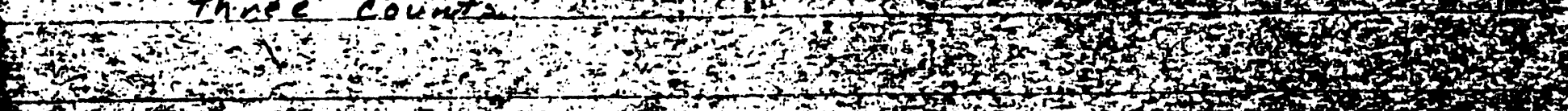

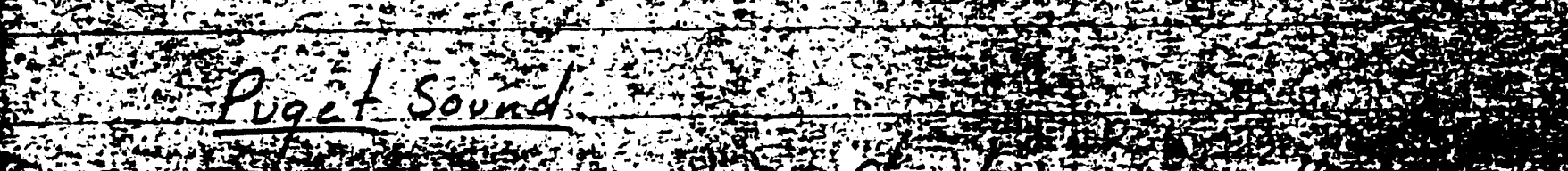

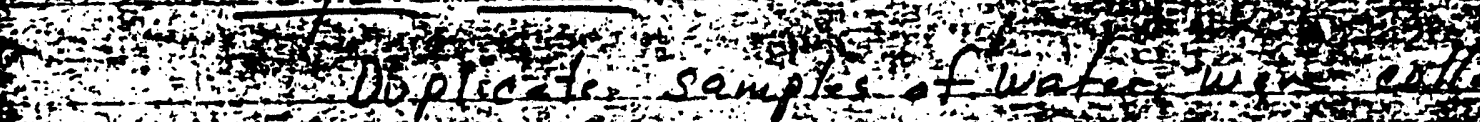

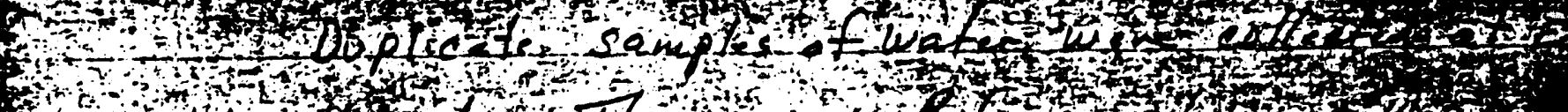

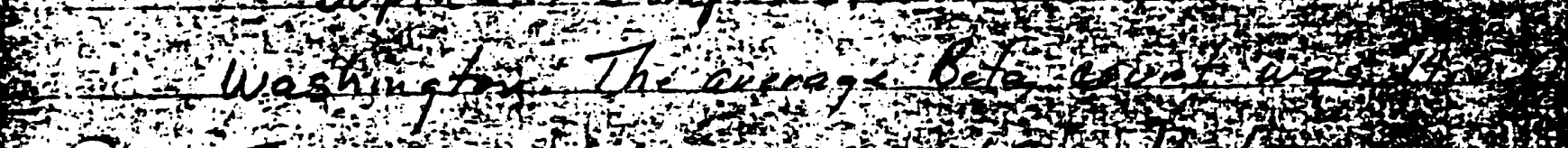

: $107 \mathrm{f}$ ave

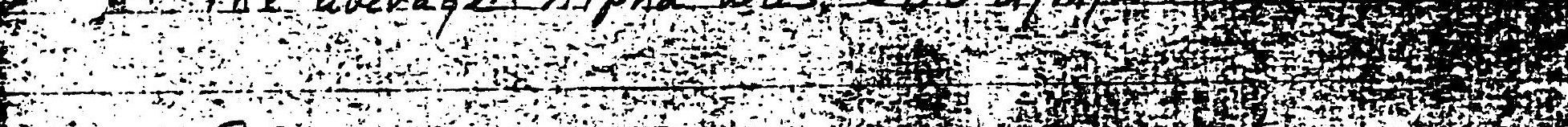

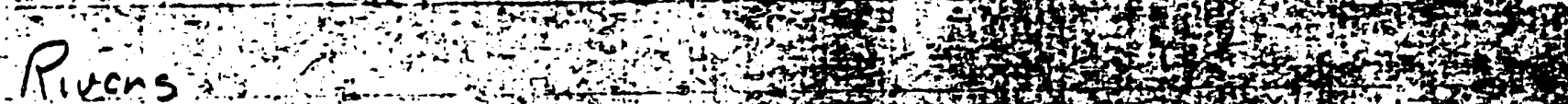

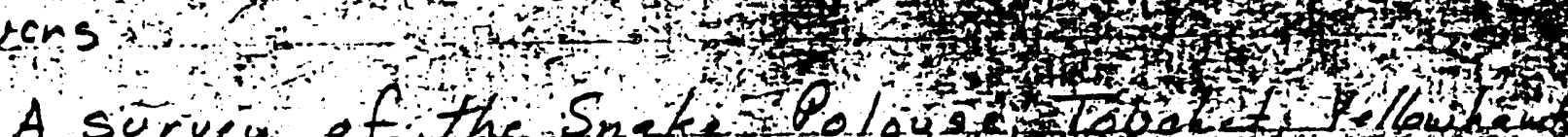

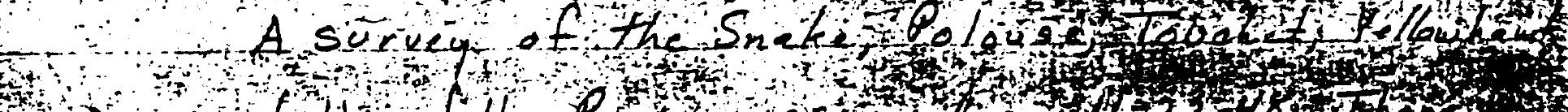

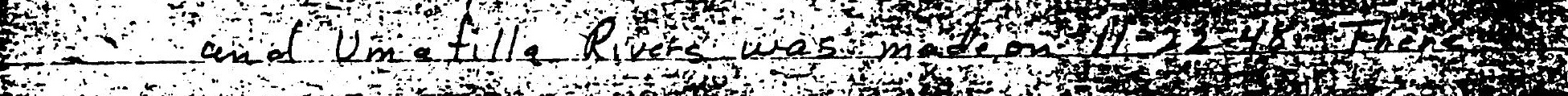

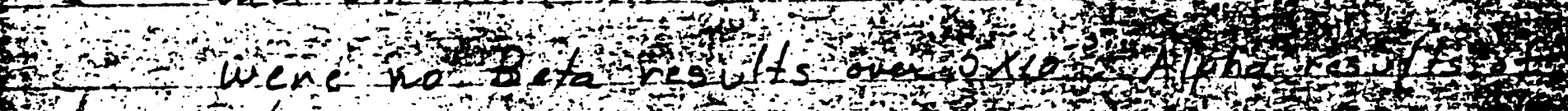

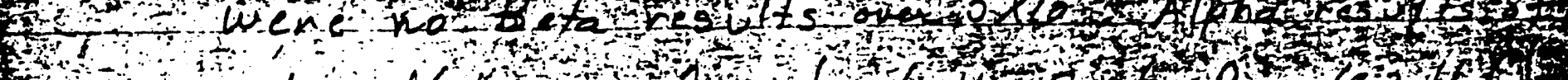

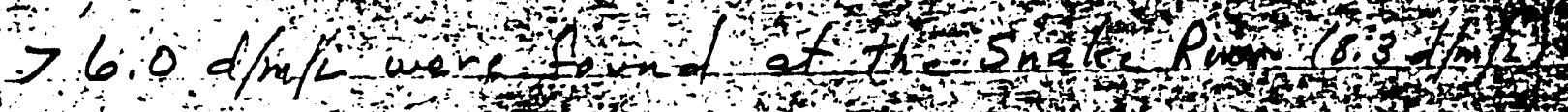

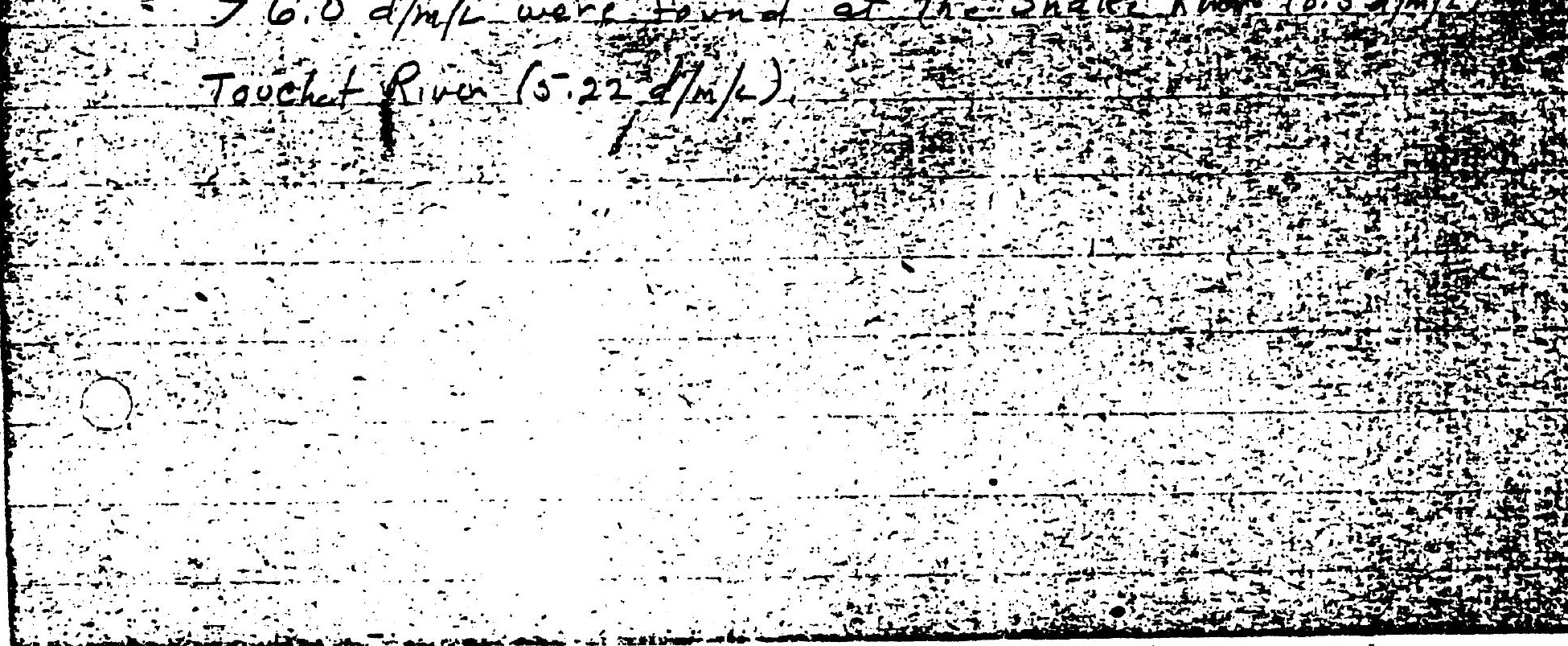




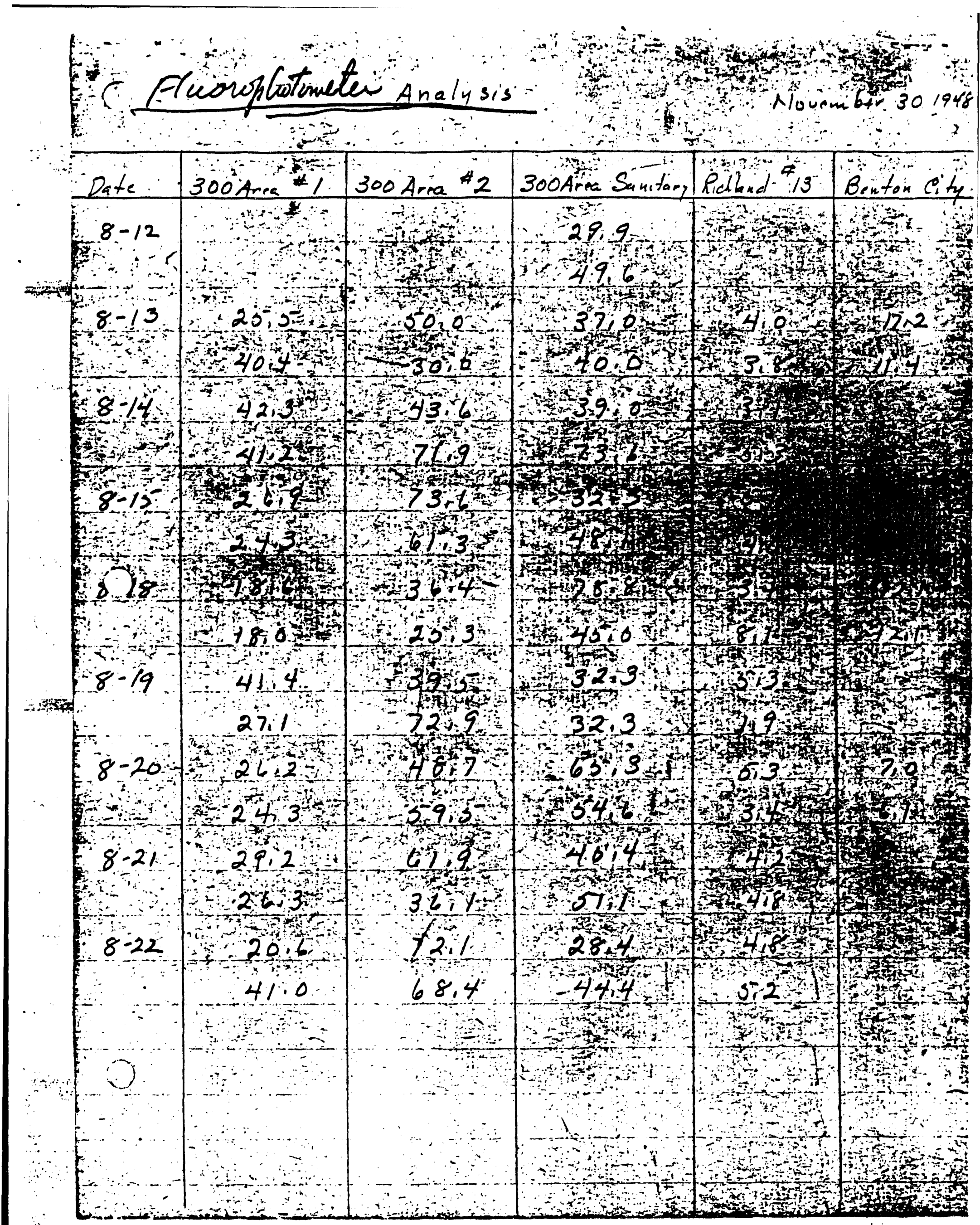




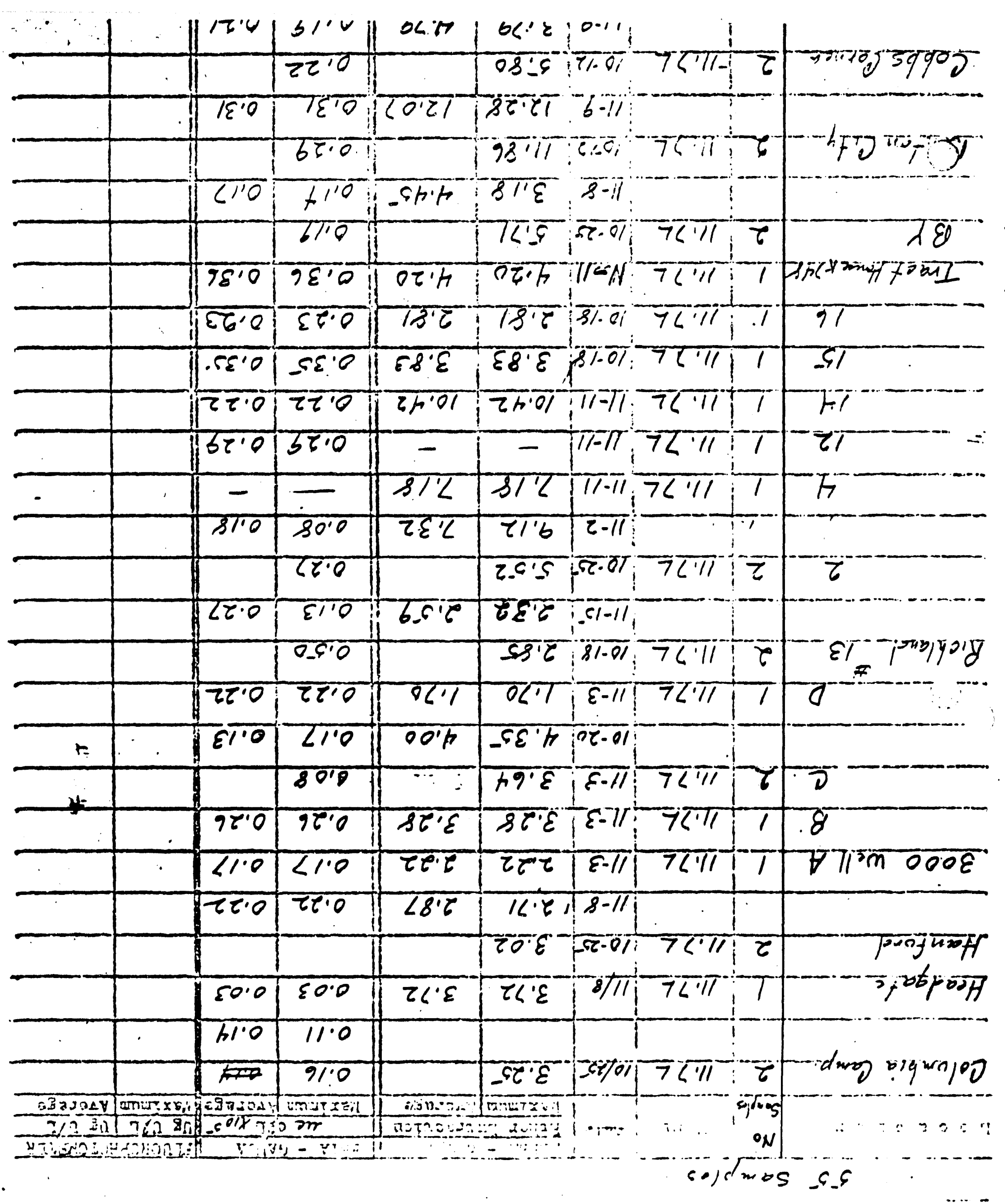

shbl'OE MqmanoN 
Page 2

C:

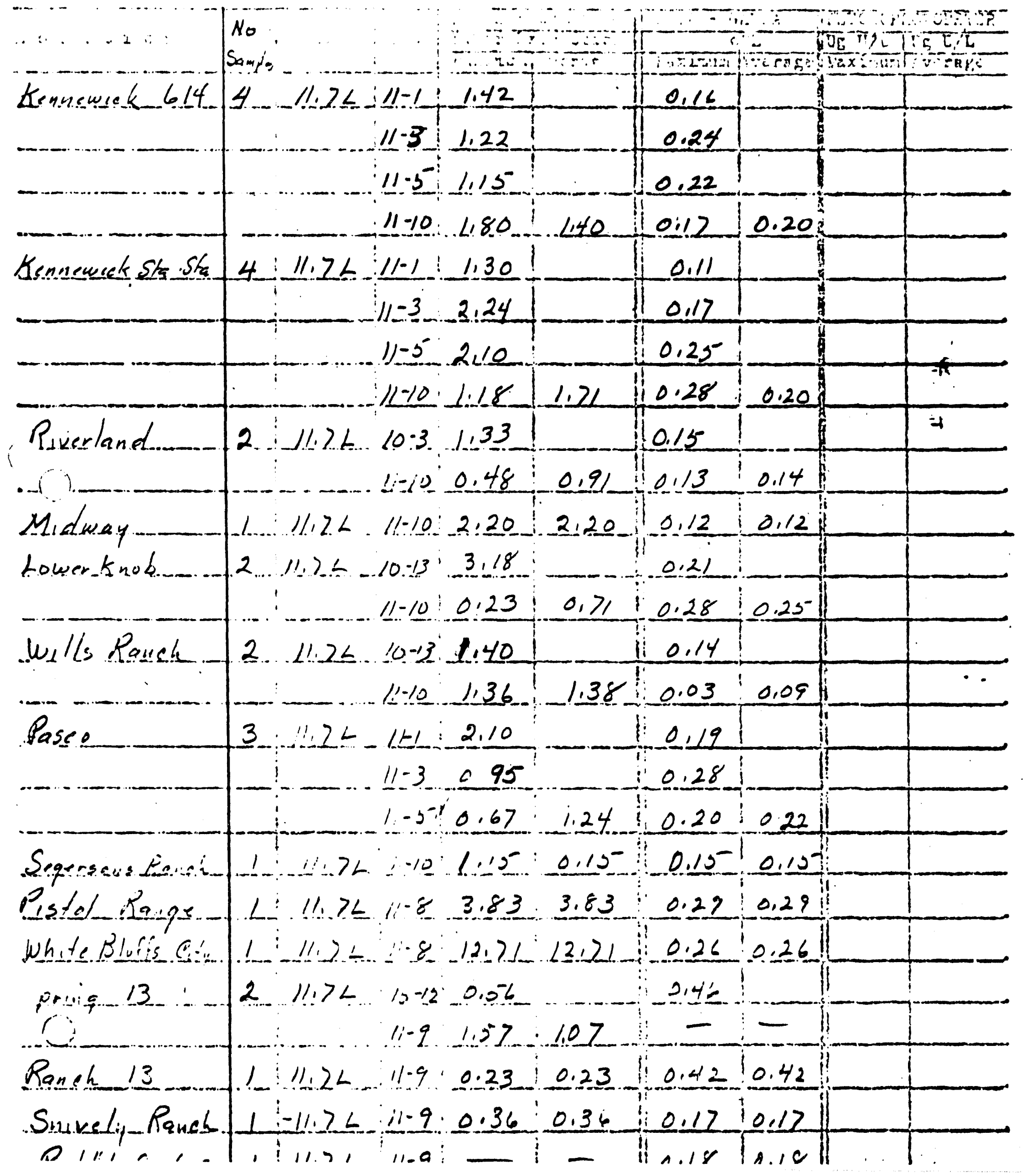




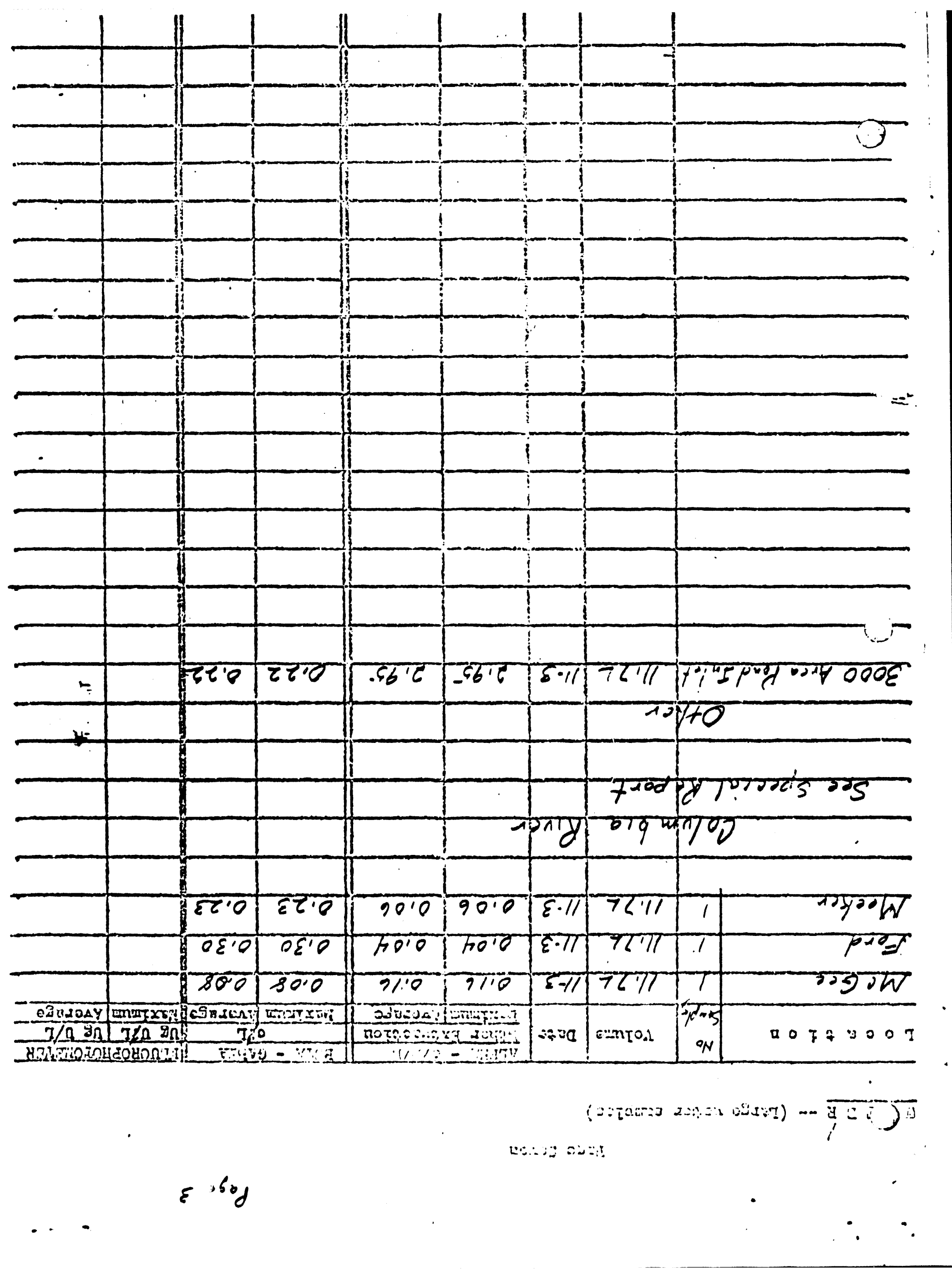


RIFER S (CORIIITD) --

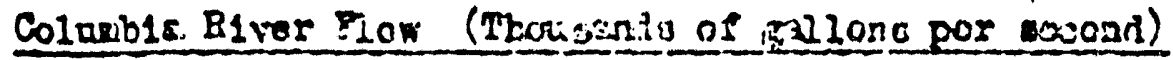

\section{Ietera Rivor -}

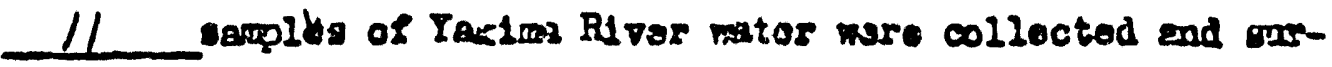
verad during uns poriod. The matemem Alphe sample wes $6.9 \mathrm{~d} / \mathrm{m} / \mathrm{t}$ talen et the mouth of the rakima' Piver. The crerazs of all eamples mas stoxef/m/m

107 Rerquont -

28_ samples of the effluent water frow the 207 badne were taken, wth recults as follows:

\begin{tabular}{|c|c|c|c|c|c|}
\hline Iopat 10a & $\begin{array}{l}\text { 10. 0x } \\
\text { Sasplee }\end{array}$ & $\begin{array}{l}\text { Alphe } \\
\text { Heximax }\end{array}$ & $\frac{a / L}{\text { Eveiraces }}$ & $\begin{array}{l}11 \text { eota end } \\
1 \text { penduse }\end{array}$ & $\begin{array}{l}\text { dened ne/L } \\
\text { drasta }\end{array}$ \\
\hline 1904.9 & 3 & $204.1^{\prime}$ & 36.9 & 0.43 & 0.36 \\
\hline & 3 & 38.8 & 13.0 & 0.30 & 0.26 \\
\hline & 3 & $69 ; 6$ & 15,1 & 0.33 & 0.22 \\
\hline $107-B$ & 6 & 50.6 & 19.1 & 0.26 & 0.24 \\
\hline$\angle 0 Z=D$ & 6 & 47,0 & 13.4 & 0.33 & 0.26 \\
\hline $107-F$ & $Z$ & 48.9 & 8.8 & 0.28 & 0.13 \\
\hline
\end{tabular}

\begin{tabular}{ll|l|l|l||l|l|}
\hline $200 \mathrm{w} \cdot$ Retention Bosio. & 8 & 51.7 & 9.1 & & $7.6 \times 10^{-5}$ \\
\hline $200 \mathrm{E} \cdot 11$ & -11 & & & & \\
\hline & & & & & \\
\hline
\end{tabular}




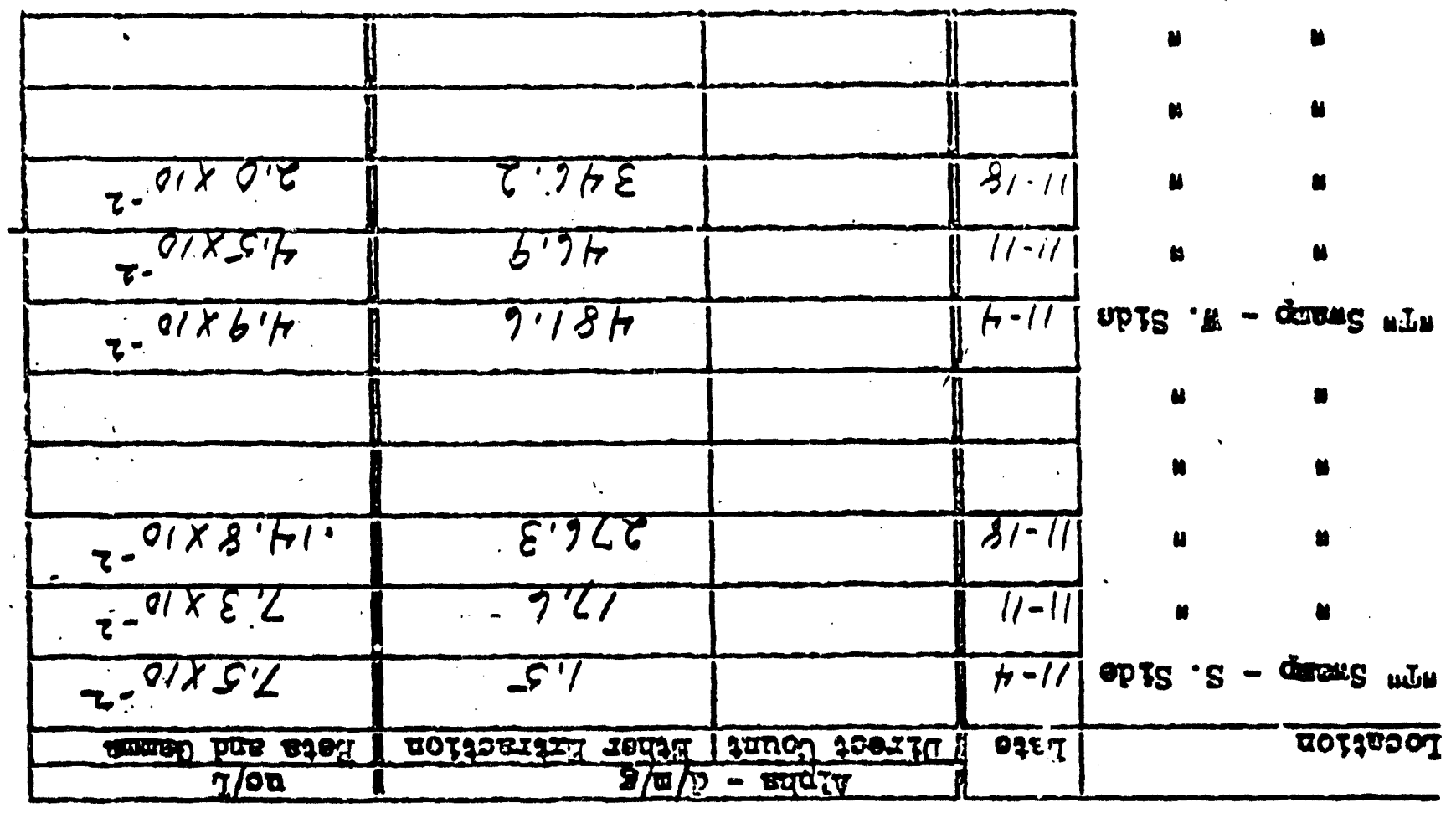

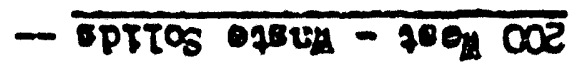

\begin{tabular}{|c|c|}
\hline$-5.01 \times 55$ & $\tau^{\prime} / \varepsilon$ \\
\hline $5=5-01 \times 56$ & 71 \\
\hline$\Leftrightarrow \varepsilon-07 \times \% \angle$ & 27688 \\
\hline$\varepsilon-01 \times b \cdot 2 T$ & T.8EF \\
\hline$: \quad 201 \times 9>$ & हण्द \\
\hline $5-0.1 \times 55$ & .6787 \\
\hline$-5.01 \times 01$ & 0.077 \\
\hline $.5-01 \times 01$ & 87 Ft \\
\hline$-5-01 \times 01$ & Z'89 \\
\hline Tlon & $I / 3 / p-84 a T y$ \\
\hline 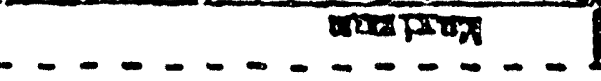 & \\
\hline
\end{tabular}

enectrapan - q8T

70Tण्I - posta lहE .009

g0tor - mosta expowi

20Tx]

opts peq - durs wh

cpts 47uog

-PTs 78ed

sotri derrins win प0178007 - BurotquTOS 97204 - 7804 COE

qrits astis 


\section{Paso 8100}

C: MASTE CONTAUINATIOB (CORTINED) 200 Rost - Raste Sol1de (Cont1 moed) --

\begin{tabular}{|c|c|c|c|c|c|}
\hline \multirow{2}{*}{\multicolumn{2}{|c|}{ Iocation }} & \multirow[b]{2}{*}{ Date } & \multicolumn{2}{|c|}{ SIpb - d $1 x$} & \multirow{2}{*}{ 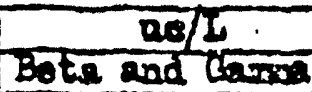 } \\
\hline & & & Dract Conat & 5thor intraction & \\
\hline Inand & int & $11-4$ & & $\therefore 287,1$ & $8.0 \times 10^{-2}$ \\
\hline 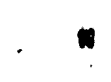 & $\omega$ & $11-11$ & & 38,5 & $3,8 \times 10^{-2}$ \\
\hline$n$ & 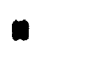 & $11-18$ & & 1334,6 & $193,6 \times 10^{-2}$ \\
\hline$\bullet$ & $\omega$ & & & & \\
\hline$\omega$ & $\bullet$ & & & & . \\
\hline
\end{tabular}

200-Biost - Portable 0. 4. Surver -

The madrum reading obtalned in this ares was $875^{-} \mathrm{c} / \mathrm{a}$ on mud Intet of tie Levidry Ditch

200 Past - Portable 0. M. Surroy -

The andmon roadios obtalned in this aros was 1700 o/n on $M$ id.

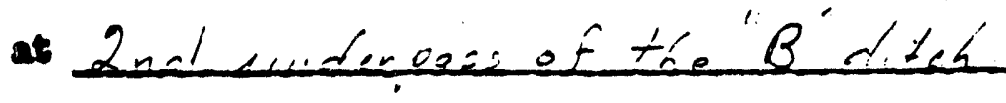




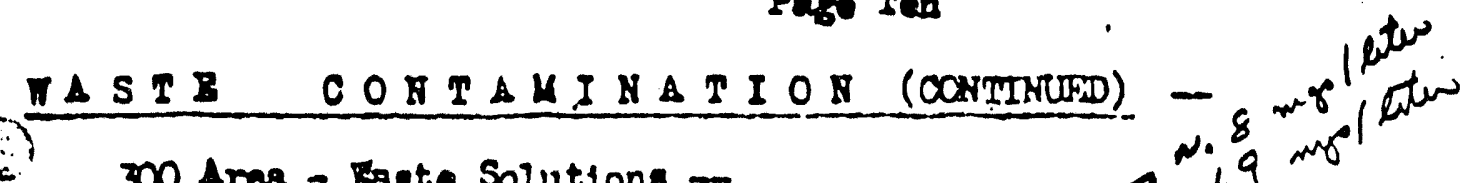

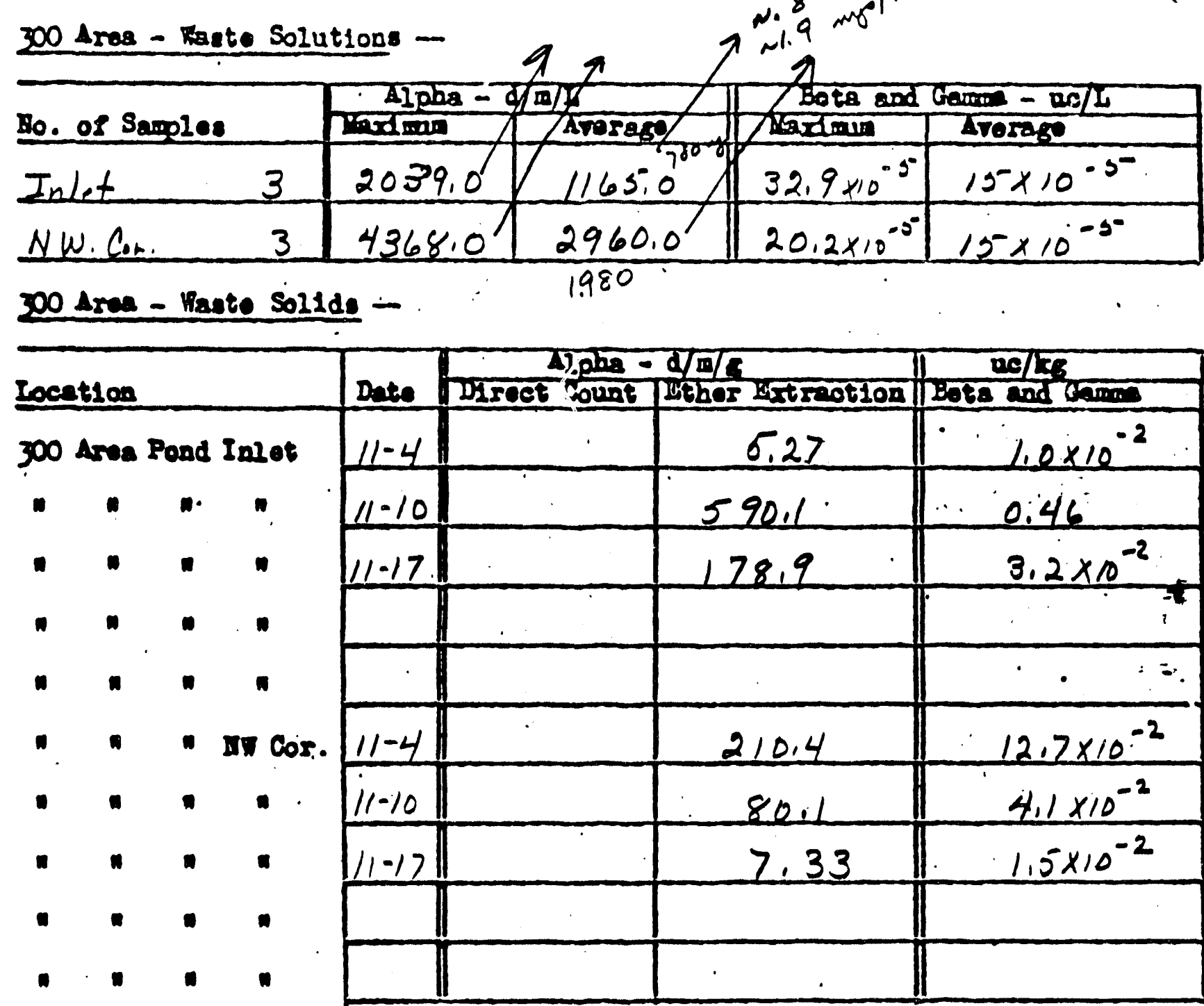

$=200$ Area - Portable O. M. Sorver -

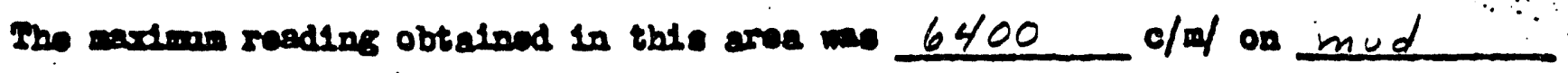
et Hi Tileter the old" 300 Area Pond.

200 Dorth Ares Fante Survere -

Ioeat1on

min Dsteh

mpn

(3)

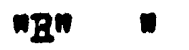
Location of Mar Rearimis
N Ditch At H.tet Puater
p.Diteh On mund at 40 fiet
$0 \wedge 1.1$ n.. an .l at..1L

Max1 14

R.ad 1 a : 


\section{O}

$\bullet$

(20)

,

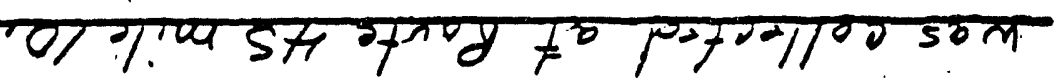

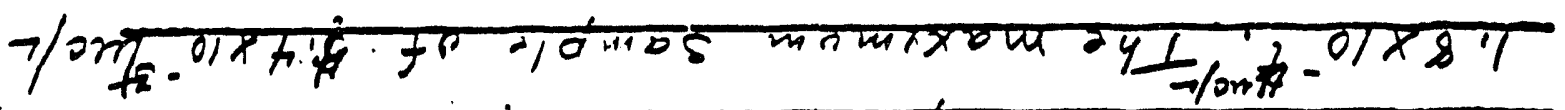

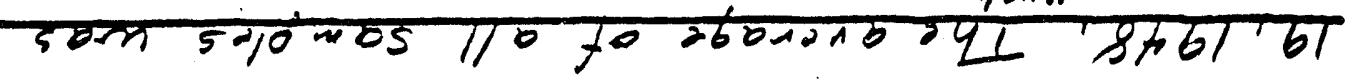

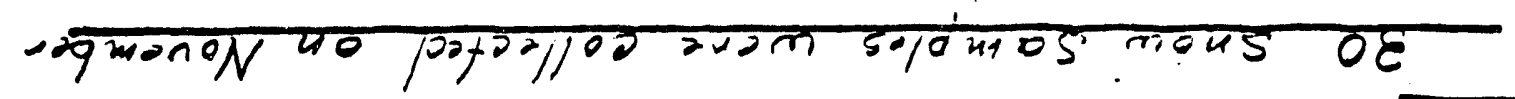

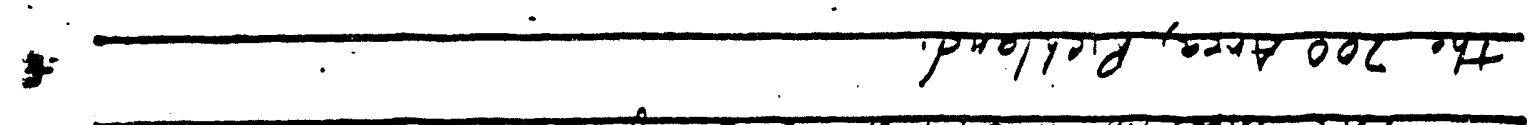
70 एकगण

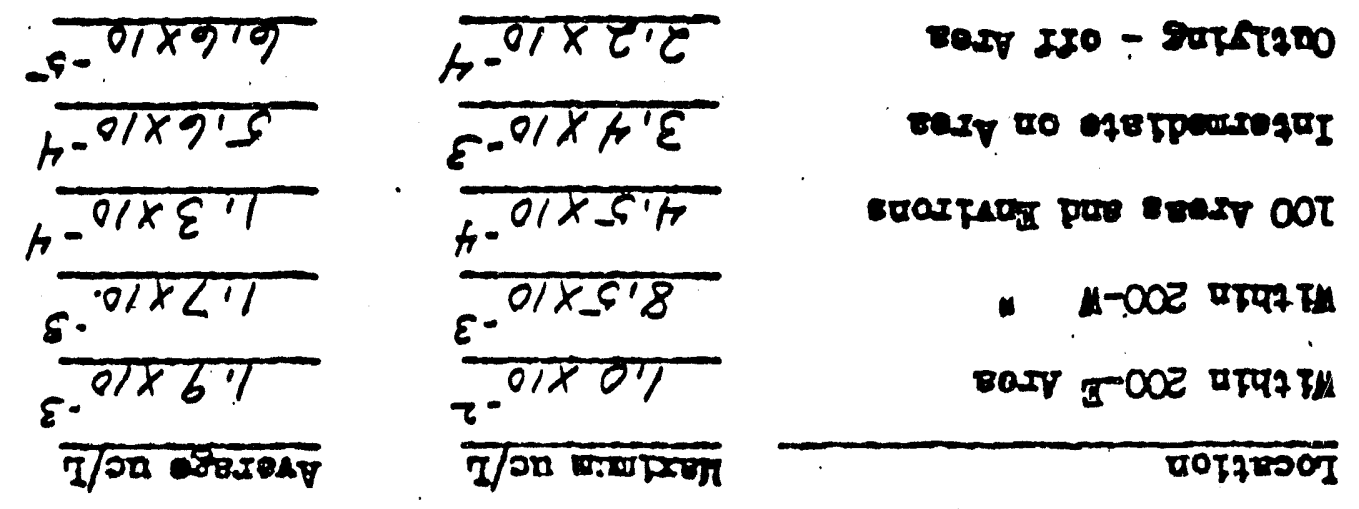

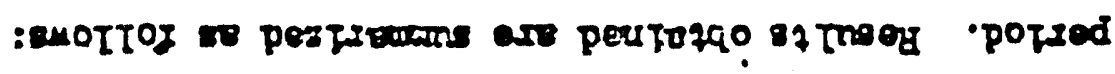

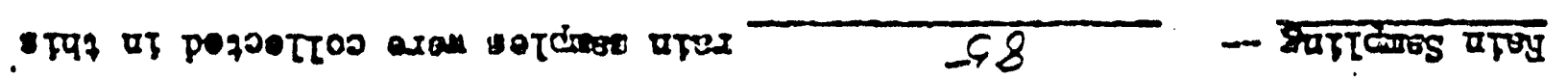

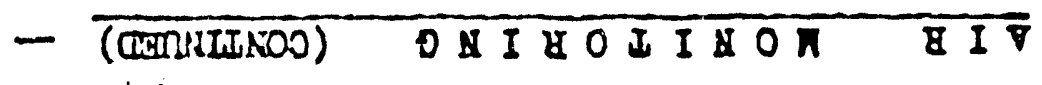

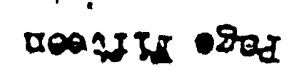



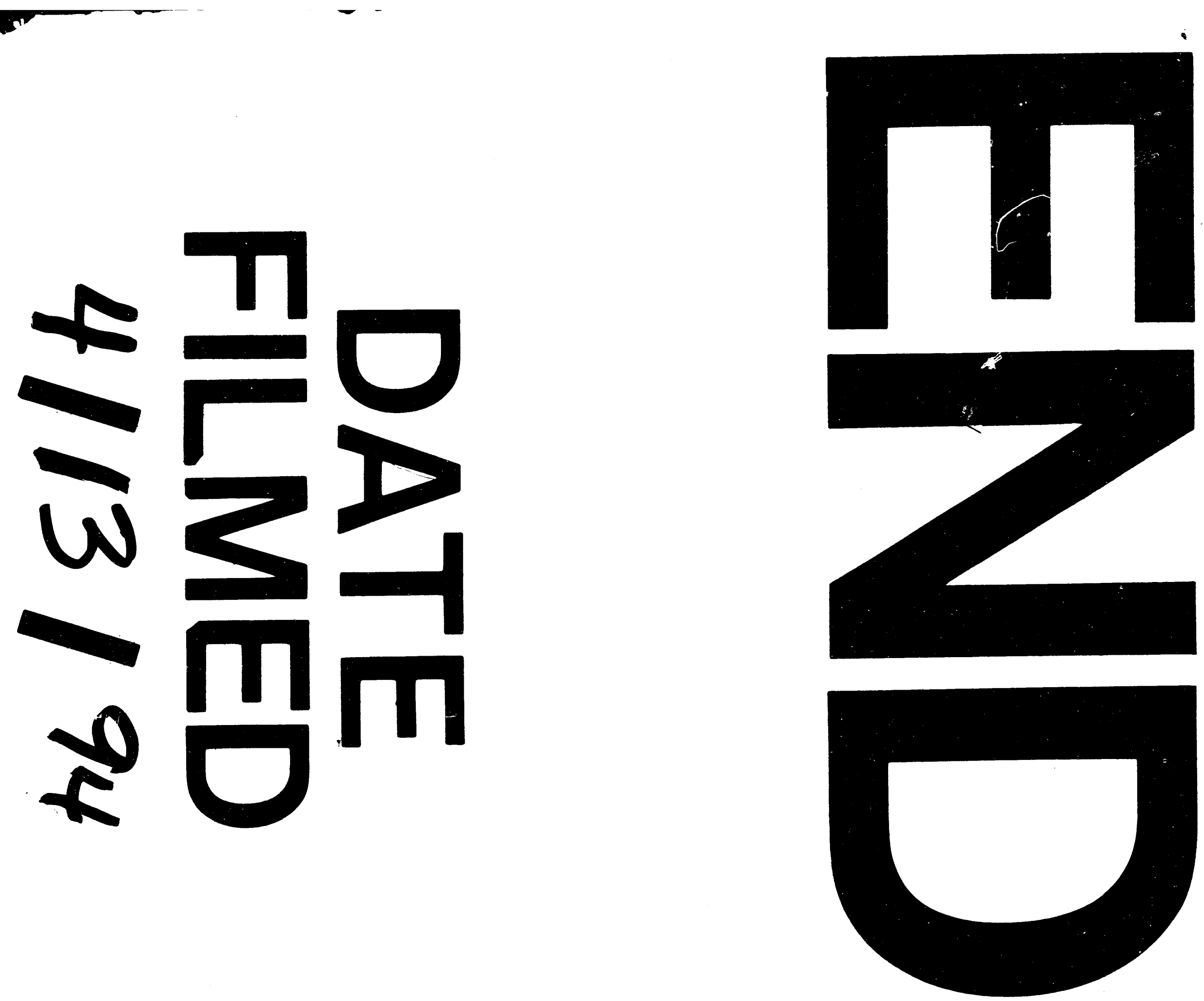

$D$ 
